\title{
A Category Theory Approach to Cognitive Development
}

\section{Author/Contributor:}

Halford, Graeme S; Wilson, William Hulme

\section{Publication details:}

Cogntive Psychology

v. 12

Chapter No. 3

pp. 356-411

0010-0285 (ISSN)

\section{Publication Date:}

1980

\section{Publisher DOI:}

http://dx.doi.org/10.1016/0010-0285(80)90014-6

\section{License:}

https://creativecommons.org/licenses/by-nc-nd/3.0/au/

Link to license to see what you are allowed to do with this resource.

Downloaded from http://hdl.handle.net/1959.4/11522 in https:// unsworks.unsw.edu.au on 2023-04-26 


\title{
A Category Theory Approach to Cognitive Development ${ }^{1}$
}

\author{
Graeme S. Halford And William H. Wilson \\ University of Queensland
}

\begin{abstract}
The category theory concept of a commutative diagram is used to construct a model of the way in which symbolic processes are applied to problem solving. The model provides for a relationship between symbolic processes and the problem which depends on structural isomorphism and consistency, but is independent of similarity between symbol elements and problem elements. It is then shown that several different levels of thought can be distinguished within the basic model. More information is needed to assign symbolic processes to a problem in a consistent way with higher-level thought processes than with lower-level processes. These information-processing requirements permit the approximate age of mastery of each level to be predicted, thereby offering an alternate theory of cognitive developmental stages. Two experiments designed to test the theory are reported.
\end{abstract}

The purpose of this paper is to offer a new approach to the definition and explanation of cognitive developmental stages. Four major stages or age groupings of cognitive development have been distinguished empirically, detailed descriptions of which are available in many general works (e.g., Flavell, 1977). The first occupies the period from birth to 1-2 years of age and has been called the sensorimotor stage by Piaget (e.g., 1950, 1953), the enactive stage by Bruner (1964), or a stage without the second signal system (Luria, 1961). This is basically a stage in which behaviour is not regulated by symbolic or representational processes.

The second main age grouping occupies approximately the period from 2 to 5 years, and has been called the preoperational period by Piaget (1950), the iconic period by Bruner (1964), a period without the "analytic system of elective significative connections which are produced by speech" (Luria, 1961), and an associative stage (White, 1965).

The period from about 5 to 10 years has been characterized by Piaget (1950) as concrete operational, as symbolic (Bruner, 1964), and as cognitive (White, 1965). The final period beginning somewhere between the ages of 11 and 15 years depending on which of a variety of criteria are adopted, has been called the formal operational stage by Piaget (e.g., Inhelder \& Piaget, 1958), and does not appear to have been redefined by any other theorist.

Despite the popularity of the stage concept among developmental theorists, the large data base which has accumulated in the last two decades has cast doubt on all existing stage theories, and even on the stage concept itself (e.g., Brainerd, 1977; Flavell, 1977; Kessen, 1962). An equally important problem is that we have no adequate theoretical basis for stages, with the result that we have difficulty accounting for even those stage-like phenomena for which we do have good evidence. The most elaborate stage theory, that of Piaget, has been shown to have serious flaws (Lunzer, 1965; Osherson, 1974; Sheppard, 1978). So long as we have no adequate, general theory of cognitive developmental stages, we cannot predict what the characteristics of each stage should be, with the result that proper empirical tests are impossible. Since there is good evidence that discontinuities in cognitive development do

1 This work was supported by grants from the Australian Research Grants Committee and from the Psychology Department of the University of Queensland. We would like to thank Murray Maybery for his excellent work, and the Queensland Department of Education for their generous cooperation. We would also like to thank several people who made valuable suggestions for the revision of this article, while reserving responsibility for the remaining deficiencies to ourselves. In particular we wish to thank Cathy Brown, Roger Brown, Bob Dick, Don McElwain, Murray Maybery, and Ray Pike.

This postprint of the paper, originally published in Cognitive Psychology 12 (1980), 356-411, corrects some typographical errors, and renames a relation in Fig. 1 in order to respect current community norms. The diagrams are re-drawn. 
occur (e.g., White, 1965), there are grounds for trying to develop a new theoretical basis for stages.

According to the approach adopted in this paper, the reason why stages exist relates to the nature of the symbolic or representational function in cognitive processes. The essence of the argument is that, since symbolic representations must be general or transferable from one situation to another, symbols must represent different objects or events in the environment on different occasions. When the problem of symbolic representation is examined, it turns out that valid representations are those which are structurally isomorphic to the particular segment of the environment to which they are applied. The achievement of isomorphic representations imposes certain information-processing demands on the organism, and these demands are greater with more complex representations. Cognitive developmental stages are postulated to occur because younger children lack the information-processing capacity to achieve isomorphic representations in the case of more complex processes. In order to develop this argument then we will first consider the nature of symbolic representation in cognition.

\section{SYMBOLIC REPRESENTATION}

One function of cognitive processes is to provide a symbolic representation of the environment as a guide to the behaviour of the organism. Representations which are used in thought must be general so that they can be applied to situations not previously experienced. Generality in this sense is defined operationally by transfer, so representations are general to the extent that they can be transferred from one situation to another.

These considerations leave us with the problem of finding a principle which governs the selection of symbols to represent objects and events in the environment. Purely "pictorial" representations, in which there is a topographical correspondence between representation and referent, are too rigid and too limited in generality to serve the functions of thought. Any principle by which the same symbol always represented the same feature of the environment would also be inadequate, because the requirement that symbols must be transferable would not be met. Therefore we need a principle which permits any symbol to represent different objects or events in the environment on different occasions, but which does not rely on purely arbitrary assignment of symbols. We will therefore examine the literature to see what solutions have been proposed for this problem.

Newell and Simon (1972) have considered the representation problem and have proposed that symbolic processes have two main constituents: symbol tokens, which are essentially what we will call symbol elements, and symbol relations. Symbol structures are built up out of symbol tokens and relations. The problem space within which the problem-solving process operates is defined by the person's internal representation of the problem. Accordingly, the representation selected must influence the problem-solving process, and we can determine what representation a person is using for a task because of the effects that it has on the actual solution processes. Newell and Simon have developed a theory of problem-solving processes which includes representations which meet the requirements we have outlined. However they do not appear to have defined a set of principles governing the operation of representations which we can use as a general criterion of representational adequacy.

One step toward the formulation of a set of principles governing representations has however been taken by Shepard, Kilpatrick, and Cunningham (1975). These authors used multidimensional scaling of similarity judgments in order to investigate the internal representation of numbers. This work was guided by the principle of second-order isomorphism, enunciated by Shepard and Chipman (1970), according to which the important 
aspect of the representational processes consists of relations between representations, rather than the representations themselves. In effect, there should be a parallelism between relations among representations and relations among the corresponding external objects.

Both this approach and that of Newell and Simon (1972) emphasize structural correspondence between representations and the corresponding external objects and events. This constitutes considerable progress toward a theory of representations, but still does not provide us with a succinct and generally applicable set of principles governing representations. It turns out however that such a set of principles is provided within the context of measurement theory (Coombs, Dawes, \& Tversky, 1970; Suppes \& Zinnes, 1963), so we will examine that formulation next.

The problem of representation in measurement theory is similar to the representation problem in cognition insofar as both are concerned with providing a valid model of the world, or of some segment of it which happens to be important for a particular purpose. In both cases a model of the world is valid if it reflects the structure of the world.

In measurement theory the structure of any aspect of the world is represented by a relational system, defined as $\left\langle A, R_{1}, \ldots, R_{n}\right\rangle$ where $A$ is a (non-empty) set and $R_{1}, \ldots, R_{n}$ are relations defined on the elements of $A$ (Coombs et al., 1970, p. 10).

The concept of a relational system is a general one and is quite applicable to cognitive psychology. In fact a theory of cognitive representations already exists which provides a clear-cut example of a relational system. Trabasso (1975) has proposed than $n$-term series (transitivity) problems are represented internally by an ordered array. For example, if participants are given five sticks varying in length, and are then told that $a>b, b>c, c>d, d$ $>e$, they encode this information in the form of an image of the five sticks arranged in order from longest to the shortest. Such an ordered set is a simple case of a relational system. The set A consists of the sticks $a, b, c, d, e$ and the relations $R_{1}, \ldots, R_{n}$ consist of the relations "larger than," "smaller than" defined on the sticks. More generally, we can think of virtually any aspect of the environment as a relational system. For instance, there are social systems consisting of organisms and their interrelations, and there are physical systems consisting of objects and their interrelations.

A particular aspect of the environment is represented if there exists a system which corresponds to it. Representations have been defined formally by Coombs et al. (1970) as follows:

a system $\alpha=\langle A, R>$ is said to be represented by another system $\beta=\langle B, S>$ if there exists a function $f$ from $A$ into $B$ (which assigns, to each $x$ in $A$, a unique $f(x)$ in $B$ ) such that for all $x, y$ in $A$

$$
x R \text { implies } f(x) S f(y) \text {. }
$$

Thus $\alpha$ is represented by $\beta$ if there exists a correspondence $f$ that maps $A$ into $B$ in such a way that if the relation $R$ holds between some $x$ and $y$ in $A$ then the relation $S$ holds between $f(x)$ and $f(y)$ in $B$, where $f(x)$ and $f(y)$ are the images of $x$ and $y$, respectively. (Coombs et al., 1970, p. 11, original authors' italics)

This definition is useful to our argument insofar as it specifies certain basic criteria which must be met before one system can be regarded as representing another. Later we will incorporate these criteria in another definition of representations which is more appropriate for use in cognitive psychology.

As applied to cognition, this definition of representation would imply that the symbolic processes of the organism must comprise systems which are in structural correspondence to the environment systems which they represent. The definition is quite general, and can apply equally to imaginal and verbal representations. Notice also that representations which 
conform to this definition need not consist of elements which resemble the corresponding environmental elements in any way. Furthermore, any element of a representation may be mapped into a number of different elements on different occasions, provided that the criterion of structural correspondence between the representational system and the environment system is observed on every occasion.

This definition appears then to have the main properties which we require in a theory of cognitive representations. In the next two sections we want to develop the argument along lines which are essentially parallel to, but eventually go beyond, those in this section. By so doing we will provide a simple and readily applicable criterion of representational adequacy of cognitive processes. We can do this by developing a theory of cognitive representations based on certain concepts from category theory, to which we will now turn.

\section{CATEGORIES}

Since category theory (Arbib \& Manes, 1975; Maclane, 1972) does not appear to have been used in psychology except for some discussions by Piaget (1970) and Piaget, Grize, Szeminska, and Vinh-Bang (1968) we will begin with the definition of a category.

Informally, a category is a collection of "objects" $a, b, c, \ldots$, and "arrows" $a \rightarrow b, \ldots$, with rules for combining the arrows. If there is more than one arrow $a \rightarrow b$, these are distinguished by labels $f_{1}: a \rightarrow b, f_{2}: a \rightarrow b$, etc. Arrows are also known as morphisms.

Formally a category $C$ consists of:

(i) a set $\mathrm{ObC}$ of objects,

(ii) for each ordered pair $(a, b)$ of objects, a set $\operatorname{Arr}_{C}(a, b)$ of arrows from $a$ to $b$, and

(iii) a composition operation: if $f: a \rightarrow b$ and $g: b \rightarrow c$ then there must exist an arrow $g_{\circ}: a \rightarrow c$.

Further

(iv) there must be, for each object $a$ an identity arrow $I_{a}: a \rightarrow a$, such that if $f: a \rightarrow b$, then $I_{b} \circ=f_{\circ} I_{a}=f: a \rightarrow b$, and

(v) if $f: a \rightarrow b, g: b \rightarrow c, h: c \rightarrow d$, then

$$
h \circ(g \circ f)=(h \circ g) \circ f: a \rightarrow d
$$

is required to hold (associativity property).

The general thrust of category theory is to describe concepts in terms of arrows rather than in terms of objects. This makes it suitable for modelling concepts which are invariant across several situations, because the set of arrows may remain constant while the objects vary, as illustrated in Fig. 1. In this case the concept of transitivity is modelled by a set of three arrows representing the relations $\mathrm{aRb}, \mathrm{Mc}$, and $\mathrm{aRc}$. The set of arrows can be applied to any transitivity problem, and two examples of such application are shown. The person's symbolic representation of transitivity is modelled by the set of arrows, which can be applied to any problem with which it is structurally isomorphic. When the representation is transferred from one problem to another, the set of arrows remains constant, but the problem elements may change completely.

A concept from category theory which is of major importance to the argument of this paper is that of the commutative diagram. This is best described by means of an example, as shown in Fig. 2. This diagram consists of objects and arrows connecting objects to at least some other objects. We can form the composites $\mathrm{v} \circ \mathrm{u}: A \rightarrow D$ and $g \circ f: A \rightarrow D$. If these are equal, we say the diagram is commutative. Less formally, we might say that the paths $(A \rightarrow C, C \rightarrow D)$ and $(A \rightarrow B, B \rightarrow D)$ must yield the same result. 


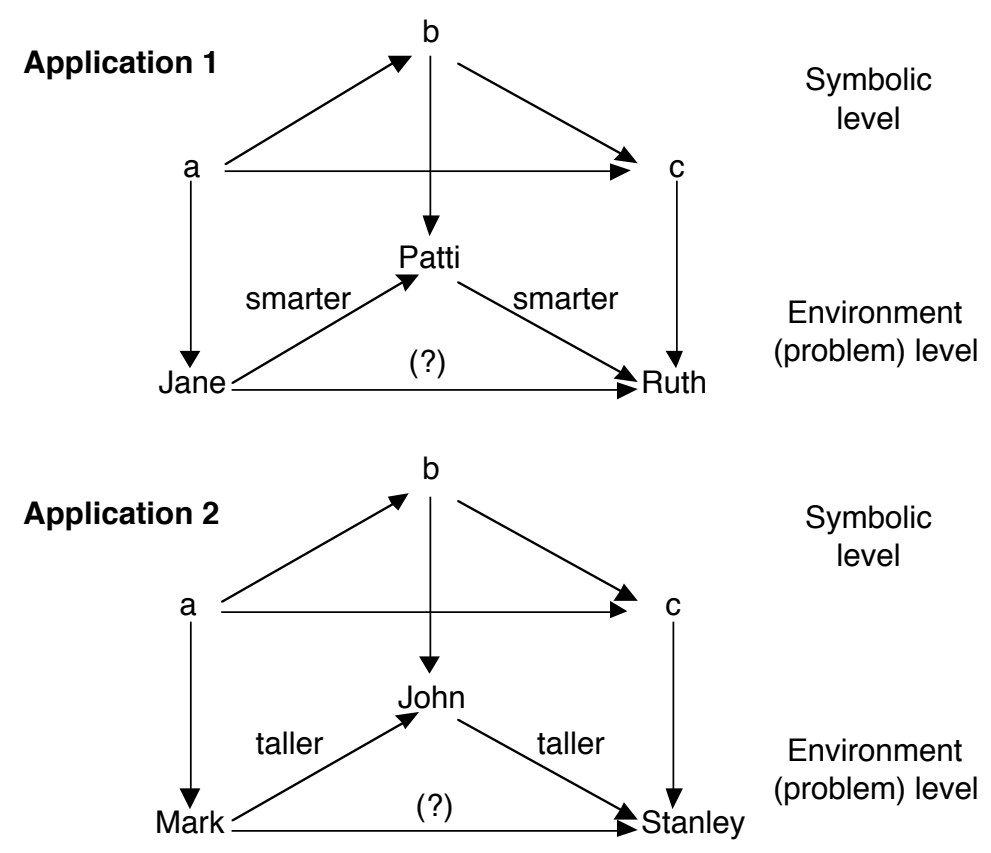

FIGURE 1 . The concept of transitivity defined as a set of three arrows, and applied to two problems in turn.

The convention used here is that a labelled arrow represents a defined set of mappings or assignments. In principle any set of mappings can be represented, but an example would be a mathematical operation such as addition. Addition of (say) integers is defined by a set of mappings of the form $\{(a, b \rightarrow c)\}$ where all $a, b, c$, are members of the set of integers; e.g., $\{(4,5 \rightarrow 9)\}$.

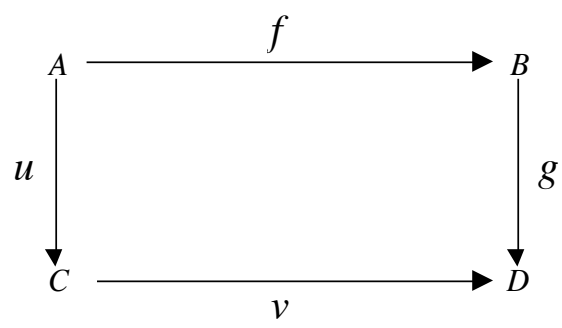

FIGURE 2. Commutativity

A single labelled arrow is employed to represent all the mappings in the set: $a, b \rightarrow^{+} c$. The label + specifies which set of mappings is intended.

Any rule, algorithm, or system can be written as a set of mappings. Thus the statement above is equivalent to asserting that an addition algorithm is a procedure for assigning ordered pairs of numbers to other numbers. The characteristics of the algorithm are defined by the mappings; e.g., since every ordered pair of numbers is assigned to one and only one number in the specified set of mappings the algorithm is one which provides a unique result to each addition problem.

The composition of arrows represents the performance of one operation followed by another.

It is important that the definition of commutativity should not be confused with its meaning in mathematical operations, where for instance $a+b=b+a$ but $a-b \neq b-a$. As the 
definition in Fig. 2 shows, the meaning of commutativity in category theory is different from the usual meaning.

\section{COGNITIVE SYSTEMS}

Now we will use the category theory concepts just outlined to construct a model of cognitive processes. Since our theory is concerned with how symbolic processes are matched to the environment in order to solve problems, we have to define symbolic processes, environment processes, and the relations between the two.

Symbolic processes are defined as symbol systems, where a symbol system is any set of internal symbolic processes which are organized in some way.

We define a set $S$ of symbols $s_{1}, \ldots, s_{n}$. We now define a symbol system as: ${ }^{2}$

$$
S \times \ldots \times S \rightarrow S
$$

This definition maps the Cartesian product $S \times \ldots \times S$ into $S$. It is equivalent to asserting that there is a process within the organism by which symbolic processes generate other symbolic processes. The arrow $f$ represents all mappings which correspond to such generative processes. Any generated symbol $S$ may be produced by varying numbers of generating symbols. Thus in an expression such as $S \times S \rightarrow S$ each generated symbol $S$ is produced by a combination of two other symbols $\mathrm{S} \times S$.

An example of a symbol system would be a person's representation of the three-term series or transitivity rule. This rule implies that given $a R b$ and $b R c$ the person can generate a symbolic expression $a R c$. In our notation this rule is simply written $a R b, b R c \rightarrow a R c$.

In order to model environment processes, we first define a set $E$ of environmental elements $e_{1}, \ldots, e_{N}$. The process by which environmental events generate other environmental events can then be written as:

$$
\begin{aligned}
& f: E \times \ldots \times E \rightarrow E \\
& f\left(e_{1}, \ldots, e_{N}\right)=d(\text { where } d \in E)
\end{aligned}
$$

This expression specifies an environment system, i.e., any organized set of events in the environment.

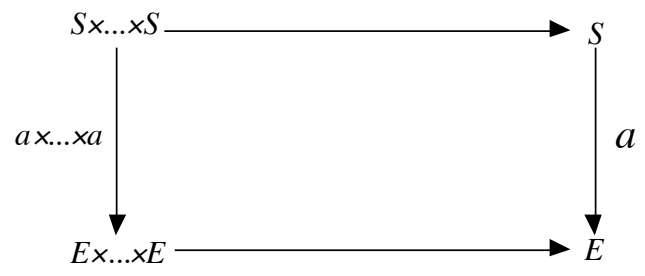

Figure 3: A Symbol System

We now define a cognitive system which is composed of a symbol system, an environment system, and the relations between the two, as shown in Fig. 3. The vertical arrows represent the application of the symbolic processes to the environment. The environment is included because insofar as environmental processes influence cognitive processes, they are part of the cognitive system. The diagram also represents varying orders of systems. That is, we could

\footnotetext{
2 Formally, a system should be defined to be the ordered pair $(S, f)$, where $f$ is as in Eq. (2), but the present notation is clearer in this context, and is always translatable into the formal notation.
} 
have a system $S \rightarrow S, S \times S \rightarrow S, S \times S \times S \rightarrow S$ etc. Each order of system belongs to a different category.

We can now define the category of all $n^{\text {th }}$ order systems:

Let us define an $n^{\text {th }}$ order system to be a set $A$ together with a function:

$$
f: A \times \ldots \times A \rightarrow A
$$

Such systems form the objects of a category $C$ whose morphisms are given as follows: a morphism $K:(A, f) \rightarrow(B, g)$ is a function $K: A \rightarrow B$ such that the diagram in Fig. 4 commutes: It can easily be verified that this construction satisfies the rules (i) to (v) for a category. We can express the cognitive system model in Fig. 3 in these terms, as shown in Fig. 5. Therefore, the set of all cognitive system models also satisfies the rules for a category.

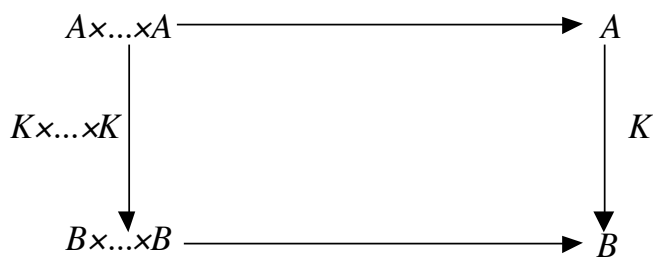

FIGURE 4

The mathematical interpretation of consistency in a cognitive system can be defined as follows. In order for an internal representation or symbol system $(S, f)$ of our environment $(E, g)$ to be consistent, we want it to be true that the processes in the symbol system exactly reflect the corresponding process in the environment system. This is interpreted mathematically by saying that there is an isomorphism of systems $i:(S, f) \rightarrow(E, g)$, that is, that there is a bijective function $i: S \rightarrow E$ such that the diagram in Figure 6 commutes. Thus consistency is expressed by a commutative diagram. (A function $i$ is bijective if it has an inverse function $i^{-1}$ such that if $a$ is mapped into $b$ by $i$ then $b$ is mapped into $a$ by $i^{-1}$. In none of the applications which follow however does the inverse function $i^{-1}$ have any implications which differ from those of $i$, and the inverse is mentioned here merely to complete the mathematical definition.)

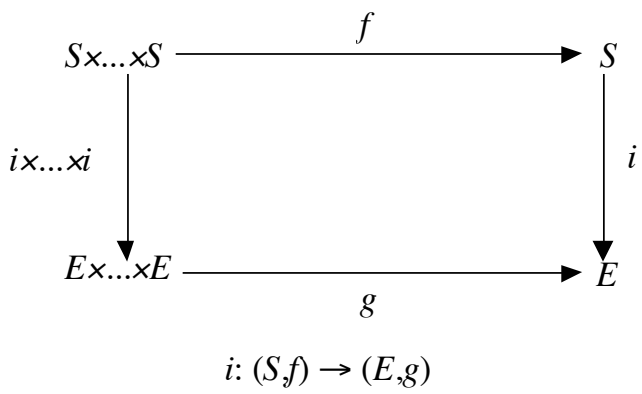

FIGURE 5

Essentially then a commutative diagram defines an appropriate application of symbolic processes to a particular segment of the environment. The commutative diagram provides a simple and fairly direct test of representational adequacy of cognitive processes, as a number of examples to be given later will illustrate.

The discussion referring to Eq. (4) and to Figs. 4-6 provides the necessary mathematical basis of our argument. Now let us consider what Fig. 3 means in psychological terms. The 
vertical arrows represent all the ways in which a particular symbol system may be applied to one or more environments. That is, the vertical arrows, like the other arrows, represent sets of mappings, and not individual mappings. This implies that a symbol element need not represent any one environment element. Thus symbolic processes do not represent specific features of the environment, but the symbols are general or transferable from one set of environment elements to another.

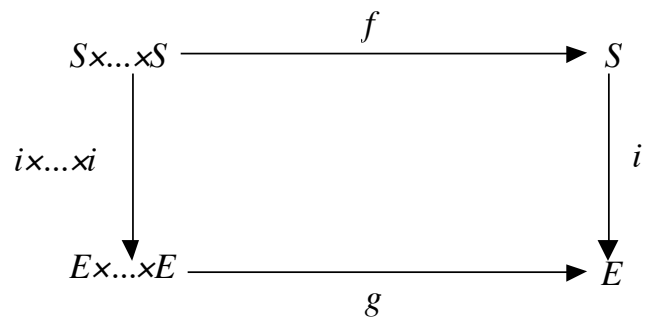

FIGURE 6

The applications of symbols to environment may vary from one situation to another, and there may even be different applications within one situation which will conform to the commutative diagram, but all mappings must be invariant within any one application.

In order to provide an example of a cognitive system diagram for a specific task, the commutative diagram for transitivity is shown in Fig. 7. The arrow $T$ specifies a set of mappings in which two relations, $a R b$ and $b R c$, are mapped into the third relation $a R c$. The actual representation used could take a number of forms, but all of them could be expressed by the set of mappings defined in Fig. 7. For instance, the triangular set of arrows in Fig. 1 can be expressed in this notation, as can the ordered array representation proposed by Trabasso (1975) for $n$-term series representations as mentioned earlier. Thus the commutative diagram model specifies a class of systems, and systems within a specific class may show some variation in the nature of the elements which they comprise. How ever, all systems belonging to a particular class would be expected to share certain common properties not shared with systems of different classes, and we will explore this aspect of the theory in the next two sections.

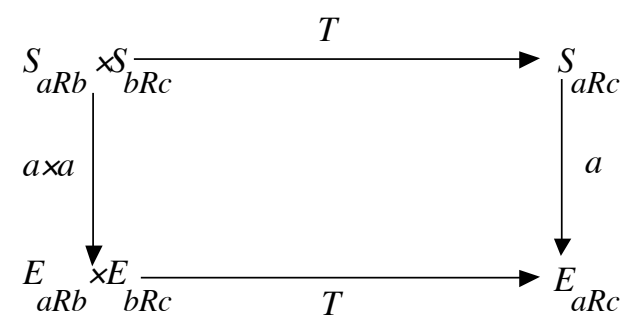

FIGURE 7

\section{ALGORITHMS, MAPPINGS, AND CONSISTENCY}

For a cognitive system to be commutative, the symbol system must be isomorphic to the environment system. This means that all mappings of symbol elements into environment elements must be invariant throughout the system. A consistency check must be made in order to insure this; i.e., a check must be made to ensure that each symbol element is mapped into one and only one environment element and vice versa. 
A question which is of importance for the argument which follows concerns the number of elements which must be checked to insure consistency. Because of the constraints which exist between elements of a system, it is not necessary to check every element.

For illustrative purposes, let us consider the number of elements which is needed to insure that the cognitive system for transitivity is commutative. The symbol system and environment system each have three elements, $a, b, c$, with relations defined on them. If we examine every case, it turns out that if $a$ and $b$ are mapped correctly, then $c$ must be mapped correctly also. Therefore the consistency check in this case needs to be based on two and only two elements.

Determinations of this kind can be made on a mathematical basis. For instance the outcome of the argument above could have been predicted simply on the basis that transitivity is a partial binary operation, and a binary operation is a system the elements of which are defined in pairs. Therefore a consistency check must be applied to pairs of elements and only to pairs.

The utility of this whole approach is that it permits us to construct arguments which are valid for classes of systems. Our convention of representing rules and algorithms by sets of mappings means that we can sort processes into equivalence classes on the basis of the form of mapping which is needed to express them. We can then construct arguments which are valid for all the processes in a particular class. In the example given in Fig. 7 we found that transitivity requires two elements to be applied for a consistency check to be carried out. Transitivity is one of the class of systems called binary operations, or more generally still, bivariate functions, and the fact that elements have to be interpreted (or applied) in pairs is true for all systems in that class. This fact in turn has implications psychologically for the information-processing load imposed on the performer.

Our next step will be to define several classes or levels of systems based on different forms of mappings. In effect, this defines a particular dimension of system complexity which we propose is related to cognitive developmental stages.

\section{LEVELS OF COGNITIVE SYSTEM}

We will define three levels of cognitive system, which differ in terms of the number of elements needed to define the system. In a level 1 system elements are defined singly; $S \rightarrow S$ or $E \rightarrow E$. In level 2 systems elements are defined in pairs, $S \times S \rightarrow S$ or $E \times E \rightarrow E$ while at level 3 elements are defined in sets of three; $S \times S \times S \rightarrow S$ or $E \times E \times E \rightarrow E$. Each level corresponds to a family of mathematical systems and also to a family of psychological tasks. We will consider each level in turn.

A level 1 cognitive system has a commutative diagram with the form shown in Fig. 8. This level of cognitive system comprises a family of mathematical concepts all of which are defined in terms of ordered pairs, and which includes binary relations, functions, and unary operators. We will define each of these in turn.

A binary relation $R$ on set $A$ is a subset $R$ of the set $A \times A$ of ordered pairs of elements of $A$. For example, if $A=\{1,2,3,4\}$ and $R$ is the relation " $>$ " or "is greater than" then $>=\{(4,3)$, $(4,2),(4,1),(3,2),(3,1),(2,1)\}$. We say $a_{1} R a_{2}$ if $\left(a_{1}, a_{2}\right) \in R$. Alternatively, a binary relation $R$ on a set $A$ can be thought of as a function $\rho: A \rightarrow \wp(A)$ where $\wp(A)$ is the power set of $A$. (The power set of the set $A$ consists of all possible subsets of $A$, including the empty set and $A$ itself.) Thus $\rho(4)=\{3,2,1\}, \rho(3)=\{2,1\}$, etc. This definition is more convenient for some of the derivations which follow.

A function from a set $A$ to a set $B$ is a set $f$ of ordered pairs $(a, b)$ (where $a \in A, b \in B$ ) such that for each element $a$ of $A$ there is precisely one $b \in B$ such that $(a, b) \in f$. It is usual to think of a function $f$ from $A$ to $B$ as a "rule" or "machine" which associates with each $a \in A$ an "output" $b \in B$. 
A unary operator $u$ on a set $A$ is a function $u: A \rightarrow A$. For example the unary operator of negation on the set of integers: $u(m)=-m$. This example should be distinguished from the binary operation of subtracting one number from another.

A unary operator is a special type of function; a function in which each element of a set is mapped into a unique element of the same set. In effect, a function from a set $A$ to a set $B$ becomes a unary operator if sets $A$ and $B$ are identical.

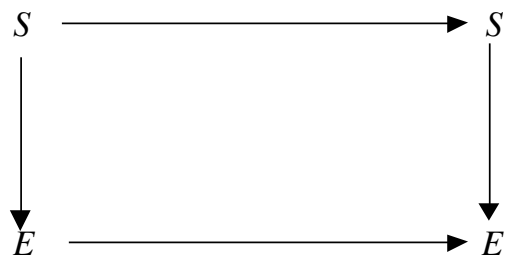

FIGURE 8

A unary operator and a binary relation on a set are related in that both consist of mappings from elements of the set to other elements of the same set. The difference is that these mappings are unique in the case of a unary operator, but not in the case of a binary relation. A function differs from both a unary operator and a binary relation in that elements of one set are mapped into elements of a second set, where the two sets may or may not be identical.

Binary relations, functions, and unary operators share a common level of complexity in that all are defined as sets of ordered pairs. Therefore we have called systems of this type level 1 and they may be defined in terms of the concepts of category theory in the following way.

Let $s, u$ denote a system which a person is using to predict or understand environment $E$ whose structure includes the unary operator $U: E \rightarrow E$. The correspondence between the objects of the system and those of the environment will be denoted by $i$ for "interpretation" in the mathematical sense, and corresponds to what psychologists would usually call "application" or "representation." The person's interpretation or representation of the environment is consistent if the diagram shown in Fig. 9 is commutative.

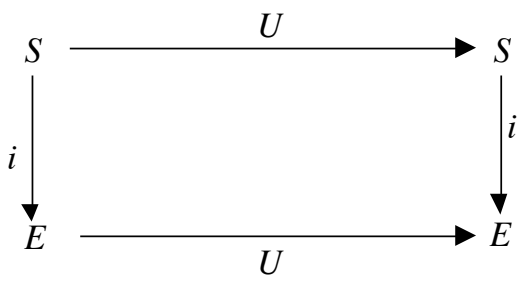

FIGURE 9

A similar diagram describes consistency for a cognitive system to represent a function. Let $f: S_{1} \rightarrow S_{2}$ be a symbol system representing an environment involving a function $g$, and let $i_{1}: S_{1} \rightarrow E_{1}$ and $i_{2}: S_{2} \rightarrow E_{2}$ be the interpretations or correspondence between the symbol and the environment. The person has consistently cognized this environment if the diagram shown in Fig. 10 is commutative.

A person's cognitive system to represent an environment whose structure corresponds to a binary relation can be expressed by defining a function from a set $A$ to the power set of $A$. Let

$$
f_{\rho, s}: A_{s} \rightarrow \wp(A)_{s}
$$

be the person's cognitive system to represent environment

$$
f_{\rho, e}: A_{e} \rightarrow \wp(A)_{e}
$$


then the person's cognition of this environment is consistent if the diagram in Fig. 11 is commutative.

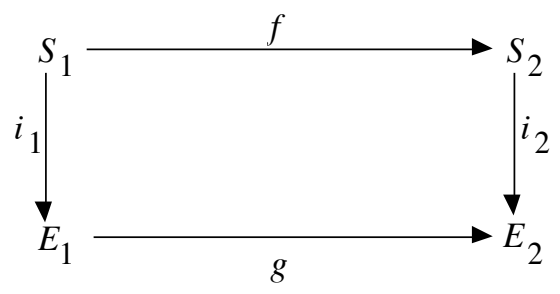

FIGURE 10

In effect all symbol systems at this level may be thought of as some kind of univariate function, or as partial univariate functions. (We note that a partial function comprises mappings of the same form as a function, the only difference being that in a partial function or operation only some mappings are defined, whereas in a function or operation all possible mappings are defined.)

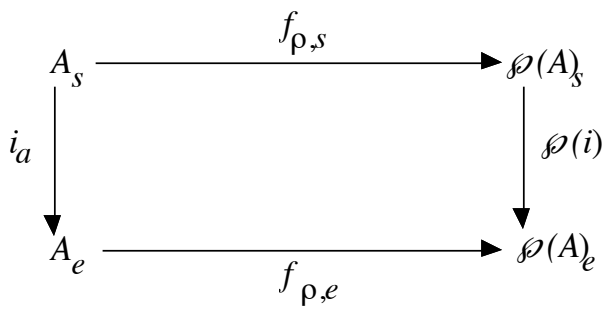

FIGURE 11

Level 2 cognitive systems are obtained when the symbol system consists of mappings of the form; $\{(S \times S \rightarrow S), \ldots\}$. Mathematically these concepts can be regarded as binary operations, binary actions, functions of two variables, and ternary relations. Every symbol system at level 2 may be thought of as a type of bivariate function or partial bivariate function, with a commutative diagram of the form shown in Fig. 12.

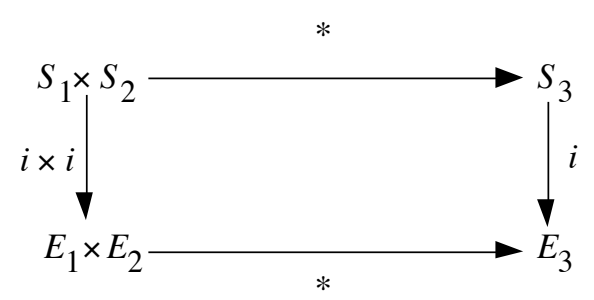

FiguRE 12

A binary operation * on a set $A$ is a function from the set $A \times A$ of ordered pairs of elements of $A$, into $A$. In arrow notation, $*: A \times A \rightarrow A$. If $\left(a_{1} a_{2}\right)$ belongs to $A \times A$, the result of applying the operation $*$ to $\left(a_{1}, a_{2}\right)$ is usually written $a_{1} * a_{2}$, and $a_{1}^{*} a_{2} \in A$. A binary action is a function $A \times R \rightarrow A$, where $R$ is a right action map. A function of two variables is a function $f: A \times B \rightarrow C$, similar to the function defined earlier, the only difference being that the set on the left of the arrow is itself a Cartesian product. The model in Fig. 7 is an example of this level of system.

At level 3 we have those symbol systems which can be defined by mappings of the form: $\{(S \times S \times S \rightarrow S), \ldots\}$. This would include compositions of binary operations, quaternary 
relations, and trivariate functions. Examples would be fields and rings, since both are structures on which the two binary operations of addition and multiplication are defined. Any cognitive system at this level could be thought of as a trivariate function or partial trivariate function, and it would be consistent if the diagram in Fig. 13 was commutative.

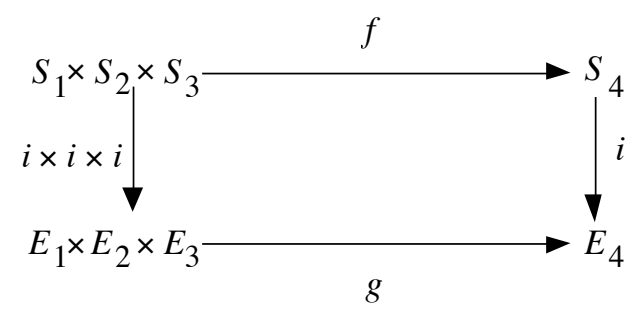

FiguRE 13

In practice, when any cognitive process is being assigned to a level, it is important that it be converted to its least complex expression; i.e., all redundancies should be eliminated. If this is done, then a level 3 system cannot be reduced to level 2, which cannot be reduced to level 1 . This is what makes the system levels mathematically distinct.

We have then three distinct levels of cognitive system, each with a different commutative diagram. More than one type of system is possible at each level and of course, there are an infinite number of possible systems of each type, but all the systems at any one level are characterized by a common level of complexity. At level 1 all symbol systems can be expressed as univariate functions, whereas at level 2 all systems must be expressed as bivariate functions, and at level 3 as trivariate functions.

\section{INFORMATION PROCESSING AND LEVELS OF COGNITIVE SYSTEMS}

Three levels of cognitive system have been defined, and it will now be shown that achievement of consistency requires more information to be processed for higher level than for lower level systems. It will be proposed that this fact imposes limitations on the level of cognitive system which children can learn at a given age. A new account of cognitive developmental stages results from these considerations.

Consistency depends on the appropriate diagram being commutative. Therefore the information which must be used to determine whether a cognitive system has been applied to a task environment consistently can be determined by examining how commutativity is ensured. The information which is required to ensure commutativity is the information which is required to ensure consistency.

In order to estimate the information required at each level, we will trace through the calculation which would be needed to test for commutativity, and record the number of separate information units or chunks which are involved at each step. This procedure will tell us not the number of chunks which an actual human participant would need to hold in store at each step, but the minimum number of chunks which is mathematically necessary. The number of chunks which a subject would require cannot however be less than the minimum which is mathematically necessary, so we will know the minimum information necessary for a human subject at each level. This is useful because it tells us the information-processing demands which would be imposed on a subject who was using the most efficient strategy which was theoretically possible. Later these findings will be related to the actual strategies which people use.

The estimation of the information-processing demand of commutativity-checking depends on the following assumptions: 
(1) Access to chunks in memory is random, not ordered. This is equivalent to saying that the subject cannot put the chunks into some kind of "push-down" store or stack which would permit him to remember only the top item on the stack. The reason is that the procedure under discussion is for use in discovering or testing algorithms, rather than in applying well-known algorithms, and there would therefore be no way of knowing how the information should be organized ahead of time. Thus whereas cognition can utilize redundancies, metacognition is concerned with the discovery of those very redundancies, and cannot use them until they are discovered, at which time it ceases to be metacognition by definition.

(2) Calculation is serial, not instantaneous.

(3) Results from earlier calculation steps which will be needed for later steps must be held in STM.

(4) The properties of the symbol system are stored in LTM. This is equivalent to asserting that the essential relationships in the problem have been learned by past experience. It does not assume however that the subject knows how to operate on the relationships so as to solve problems. This distinction is operationalised in the experiments to be reported later in this paper.

The psychological validity of these assumptions will be considered later.

Some prior explanation is needed about the calculations presented below. A check for commutativity requires that we begin with the element on the top left of the diagram, then proceed first by one path and then the other to the element on the bottom right of the diagram. If the results obtained by each of the two paths are the same, the diagram is commutative; otherwise it is not. To make the presentation of the calculation clearer, we will number the vertices of the diagram as follows: 1 is at the top left, 2 at the top right, 3 at the bottom left, and 4 at the bottom right. Each step in the calculation involves converting elements at one vertex into elements at the next. Information from any step which must be used for later steps is held in store (Assumption 3), so the information which would be in temporary store at each vertex is shown. The number of chunks in store at each vertex can be determined, and the maximum information load can be read off.

The commutativity calculation can be made in two ways, depending on which path is taken first. Starting at Vertex 1 of the commutative diagram (element $S$ for the level 1 diagram below) we can proceed vertically to $i(S)$ then horizontally to $u(i(S))$. (Here $i(S)$ is the element which results from interpreting $S$, and $u(i(S))$ is the element which results from operation of the environment system on $i(S)$.) Alternatively we can proceed horizontally from $S$ to $u(S)$, then vertically to $i(u(S))$. These procedures, which we call Method 1 and Method 2, respectively, exhaust the methods which involve proceeding in the one direction until the end of that path is reached; i.e., they exhaust all calculation procedures which involve passing through each vertex other than the first and last once only. The calculation will be made in both ways even though in some cases the result is the same.

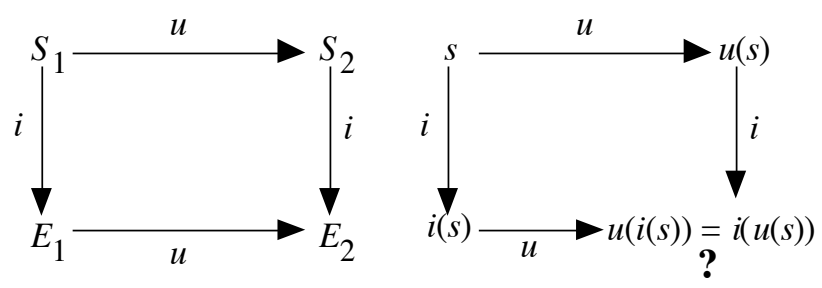

FIGURE 14

For level 1 we have the commutative diagrams shown in Fig. 14, the more general form of the expression being that on the right. For commutativity to hold at level $1, u(i(S))$ must be 
equal to $i(u(S))$. The check for commutativity involves calculating each of these. The steps in Method 1 are as follows.

\begin{tabular}{clc} 
Vertex & $\begin{array}{l}\text { Information in } \\
\text { temporary store }\end{array}$ & Number of chunks \\
\hline 1 & $S$ & 1 \\
3 & $i(S), S$ & 2 \\
4 & $u(i(S)), S$ & 2 \\
2 & $u(i(S), u(S)$ & 2 \\
4 & $u(i(S), i(u(S))$ & 2
\end{tabular}

This method interprets the symbols first, then computes the result corresponding to the operation or action map. However the second method reverses the order of these steps.

\begin{tabular}{clc} 
Vertex & $\begin{array}{l}\text { Information in } \\
\text { temporary store }\end{array}$ & Number of chunks \\
\hline 1 & $S$ & 1 \\
2 & $u(S), S$ & 2 \\
4 & $i(u(S)), S$ & 2 \\
3 & $u(i(S), i(S)$ & 2 \\
4 & $u(i(S), u(i(S))$ & 2
\end{tabular}

With level 1 systems these two methods differ only trivially, and the peak information load, two chunks, is the same for both methods. However this is not the case at the higher levels as we will see.

For level 2 we have the commutative diagrams shown in Fig. 15. For commutativity to hold at level 2, $i\left(S_{1}\right) * i\left(S_{2}\right)$ must equal $i\left(S_{1} * S_{2}\right)$. The steps in Method 1 are as follows.

\begin{tabular}{clc} 
Vertex & $\begin{array}{l}\text { Information in } \\
\text { temporary store }\end{array}$ & Number of chunks \\
\hline 1 & $S_{1}, S_{2}$ & 2 \\
3 & $i\left(S_{1}\right), i\left(S_{2}\right), S_{1}, S_{2}$ & 4 \\
4 & $i\left(S_{1}\right) * \mathrm{i}\left(S_{2}\right), S_{1}, S_{2}$ & 3 \\
2 & $i\left(S_{1}\right) * \mathrm{i}\left(S_{2}\right), S 1 * S 2$ & 2 \\
4 & $i\left(S_{1}\right) * \mathrm{i}\left(S_{2}\right), i\left(S_{1} * S_{2}\right)$ & 2
\end{tabular}

Here the peak information load is 4 chunks. Now using Method 2, the steps are as follows.

\begin{tabular}{clc} 
Vertex & $\begin{array}{l}\text { Information in } \\
\text { temporary store }\end{array}$ & Number of chunks \\
\hline 1 & $S_{1}, S_{2}$ & 2 \\
2 & $S_{1} * S_{2}, S_{1}, S_{2}$ & 3 \\
4 & $\mathrm{i}\left(S_{1} * S_{2}\right), S_{1}, S_{2}$ & 3 \\
3 & $\mathrm{i}\left(S_{1} * S_{2}\right), i\left(S_{1}\right), i\left(S_{2}\right)$ & 3 \\
4 & $\mathrm{i}\left(S_{1} * S_{2}\right), i\left(S_{1}\right) * i\left(S_{2}\right)$ & 2
\end{tabular}

Here the peak information load is 3 chunks, so at level 2 the two methods produce different information-processing demands. 
At level 3 the commutative diagrams are as shown in Fig. 16. For commutativity to hold at level 3, $f\left(i\left(S_{1}\right), i\left(S_{2}\right), i\left(S_{3}\right)\right)$ must equal $i\left(f\left(S_{1}, S_{2}, S_{3}\right)\right)$. The steps in Method 1 are as follows.

\begin{tabular}{clc} 
Vertex & $\begin{array}{l}\text { Information in } \\
\text { temporary store }\end{array}$ & Number of chunks \\
\hline 1 & $S_{1}, S_{2}, S_{3}$ & 3 \\
3 & $i\left(S_{1}\right), i\left(S_{2}\right), i\left(S_{3}\right), S_{1}, S_{2}, S_{3}$ & 6 \\
4 & $f\left(i\left(S_{1}\right), i\left(S_{2}\right), i\left(S_{3}\right)\right), S_{1}, S_{2}, S_{3}$ & 4 \\
2 & $f\left(i\left(S_{1}\right), i\left(S_{2}\right), i\left(S_{3}\right)\right), f\left(S_{1}, S_{2}, S_{3}\right)$ & 2 \\
4 & $f\left(i\left(S_{1}\right), i\left(S_{2}\right), i\left(S_{3}\right)\right), i\left(f\left(S_{1}, S_{2}, S_{3}\right)\right)$ & 2
\end{tabular}

Here the peak information load is 6 chunks. Now using Method 2, the steps are as follows.

\begin{tabular}{|c|c|c|}
\hline Vertex & $\begin{array}{l}\text { Information in } \\
\text { temporary store }\end{array}$ & umber of chunks \\
\hline 1 & $S_{1}, S_{2}, S_{3}$ & 3 \\
\hline 2 & $f\left(S_{1}, S_{2}, S_{3}\right), S_{1}, S_{2}, S_{3}$ & 4 \\
\hline 4 & $i\left(f\left(S_{1}, S_{2}, S_{3}\right)\right), S_{1}, S_{2}, S_{3}$ & 4 \\
\hline 3 & $i\left(f\left(S_{1}, S_{2}, S_{3}\right)\right), i\left(S_{1}\right), i\left(S_{2}\right), i\left(S_{3}\right)$ & 4 \\
\hline 4 & $i\left(f\left(S_{1}, S_{2}, S_{3}\right)\right), f\left(i\left(S_{1}\right), i\left(S_{2}\right), i\left(S_{3}\right)\right)$ & 2 \\
\hline
\end{tabular}

Here the maximum information-load is four chunks.
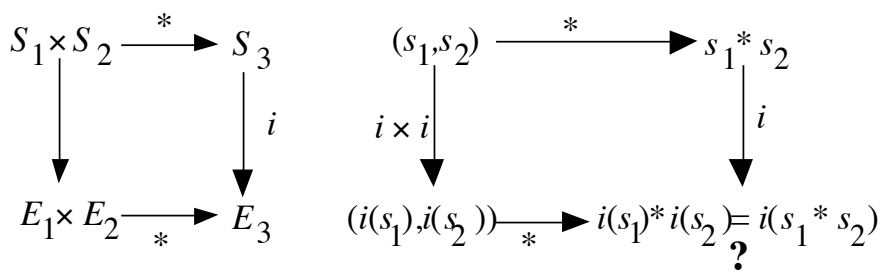

FigURE 15

It is clear from this exercise that, provided the method of calculation is level independent, the information load is always higher for higher-level systems than for lower-level systems. This is inevitable because of the fact that level 1 systems are actually defined by a single variable (in the sense that any level 1 system can be expressed as some kind of univariate function), level 2 systems are defined by two variables (bivariate functions), and level 3 by three variables. Therefore it is inevitable that more information will be needed to check the consistency of application of a higher-level symbol system to the environment than a lowerlevel system.
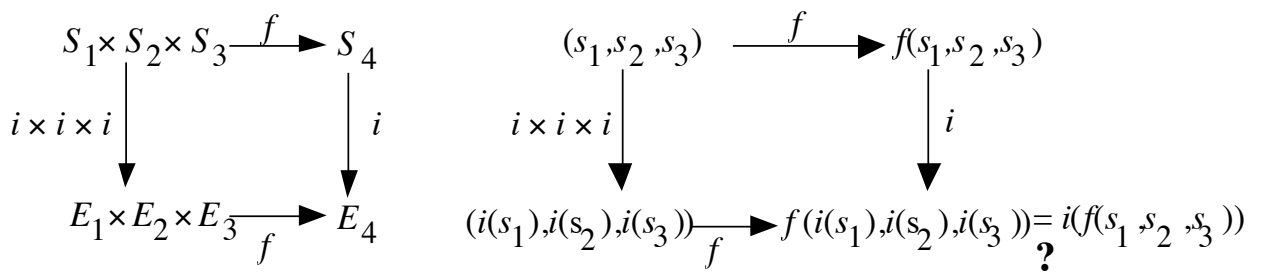

FIGURE 16 
To summarize the argument so far, we have defined a symbol system as an organized set of elements which is internal to the organism. An environment system is an organized set of elements in the environment. A symbol system represents an environment system if the former can be mapped into the latter so that the resulting cognitive system conforms to a commutative diagram. We have defined three levels of cognitive systems, and have shown that more information needs to be processed to insure commutativity of higher-level cognitive systems than for lower-level cognitive systems. Our next step is to relate this argument to the performance of human organisms performing cognitive tasks. In the next section we will consider where the symbol system is stored, and the processes which are involved in mapping the symbol system into the environment system so as to form a commutative cognitive system.

\section{COGNITIVE PROCESSES AND MEMORY STORAGE}

A person's representation of a problem consists of a symbol system, as we have already stated, and such a system must be stored somewhere in memory. Certain considerations make it seem likely that a symbol system would be stored in long-term memory (LTM). First, problem solving is often a protracted process, and since the symbol system contains the person's representation of the problem it would need to be continuously available. Second, if symbol systems are stored in long-term memory (LTM) this would leave short-term memory $(S T M)$, or working memory, free for the problem-solving task. Third, symbol systems may be used for a number of problems in succession, perhaps spread over a considerable time, and storage in $L T M$ would make them available for this purpose.

On the other hand it is unlikely that the actual data of a new problem are stored in LTM. Klahr and Wallace (1976) considered this possibility in the context of certain cognitive developmental tasks and concluded that the problem-solving process was too fast to permit transfer to LTM. There are exceptions. For instance, Bryant and Trabasso (1971) systematically taught the comparisons of a $n$-term series problem to children, so that the actual relations between the stimuli, $a>b, b>c$, etc. were stored in LTM. Such a procedure is unusual however in a problem-solving context, where one or two trials only are normally used. In general, we can say that the actual problem elements are not stored in LTM.

It would follow from these considerations that when a problem is presented the problem elements and relations are not stored in $L T M$, but a structurally isomorphic situation in LTM is used to serve as a model of the problem. This is made possible by the fact that the symbol system need not contain elements which resemble those in the problem. Structural correspondence is all that is required, which means that any problem can, in principle at least, be represented by any structurally isomorphic system stored in LTM. For example, a transitivity problem could be represented by any three objects from the past experience of the problem solver which happen to form an ordered set.

The whole problem-solving process cannot however take place in LTM because, as we pointed out before, problem solving must entail dealing with new situations. Any process which cannot draw on LTM is therefore likely to draw on STM. That is, STM is the work space within which those aspects of problem solving which are essentially new are carried out, but this process draws on representations of the problem stored in LTM. This argument is essentially similar to the proposals of Greeno (1973), Hunt (1978), and Klahr and Wallace (1976) that problem solving draws on STM but utilizes data stored in LTM.

One aspect of problem solving which is likely to draw on STM is the assignment of a symbol system to a new problem. Where the same representation has been repeatedly applied to a particular problem, an algorithm for assigning the symbol system to the environment 
system may be acquired and stored in LTM, but this would not be the case when a new problem was being attempted. The situation where a child first makes the transition from one level of thought to another would inevitably be of the kind where the assignment of symbol system to environment system draws on STM.

This suggests that STM limitations may well impose constraints on the process of applying a symbolic representation or symbol system to a new problem. Since higher-level systems impose higher information processing requirements, this may limit the highest level of system with which children can operate. Since we have a set of estimates of the number of chunks of information which must be used when assigning a symbol system to an environment system, we may be able to predict the highest level of system with which children can operate from STM considerations.

One question which needs to be considered first is whether the assignment of symbol system to environment system is the only process which would draw on STM. We are mainly concerned with predicting the age of transition to a new level, and it seems likely that this transition would first be made in the most familiar situations. In such cases all performance aspects of the task would probably be well learned, and would therefore be stored in LTM, so they would make little or no demand on STM. In order to predict the earliest point at which a given level of cognitive system could be developed in any context, it would therefore seem most appropriate to assume that task performance makes no demand on STM. This permits us to assume that the whole of STM capacity is available for the process of assigning a symbol system to an environment system.

The argument above implies that we should be able to predict the earliest age at which children could construct a cognitive system at any given level if we can measure the shortterm memory capacity which children have at each age. One difficulty in making this prediction is that we in effect have one free parameter in our estimates of the number of "chunks" of information required for a given level. This parameter corresponds to the distinction between Method 1 and Method 2 in the previous section. However, we can make two sets of predictions, one for each method, then test to see which method yields the best predictions. If the same method is consistently valid, we will in effect have estimated this parameter from the data.

There is little doubt that performance on STM tasks increases with age, but there is widespread disagreement as to the nature of the underlying processes which yield this increase. On the one hand it is argued that the increase is due, not to an underlying increase in capacity, but to the development of better mnemonic strategies (e.g. Flavell, 1971; Dempster, 1978). A slightly different view is presented by Simon (1974) who suggests that the number of chunks remembered is constant over age, but chunk size increases. Although we are perfectly willing to accept the evidence that children develop better mnemonic strategies as they grow older, this does not of itself argue that capacity does not increase as well. In those studies where strategies have been controlled, capacity differences related to age and intelligence have been found (Cohen \& Sandberg, 1977; Friedrich, 1974; Huttenlocher \& Burke, 1976; Lyon, 1977). A further point is that the mnemonic strategies which have been found to occur in recall tasks may not be applicable in problem solving, as pointed out by Hunt (1978).

In order to predict ability to construct cognitive systems of level $n$, we need to know how many chunks can be held in short term store. According to Miller (1956) adults can hold $7 \pm 2$ chunks, but Broadbent (1975) suggests the number is really 3. Broadbent does seem to suggest however that there are two types of short-term memory, each of which can hold 3 or possibly 4 chunks, so that the capacity of adult $S T M$ would be functionally equal to about 7 chunks. 
While recognizing that $S T M$ itself is an immensely complex and as yet little-understood phenomenon, in this argument we are really only concerned with the functional size of STM. That is, we are only concerned with the number of chunks which a person can hold in shortterm store at one time, irrespective of the precise mode of storage. For our present purposes, it is sufficient to define a chunk as an independent item of information which may vary in size; i.e., two signals cannot be combined into one chunk if they vary independently of each other.

The traditional memory span test, such as the digit span tests used in psychometric intelligence tests, probably provides a fairly good approximate measure for this purpose. The digits are presented in a way which is independent according to our definition, because runs or other sequential constraints are eliminated as far as practicable. This task also has the advantage that age norms are available, which is not yet the case for the more esoteric measures. The digit span test can be regarded as measuring functional STM capacity, for ordered sets of items. It may seem inappropriate to use memory for ordered items in order to predict ability to construct cognitive systems, because the information required to check the consistency or commutativity of such systems is not ordered. On the other hand, the information required for commutativity checking is tagged, in the sense that each item of information is distinguished from the others. Therefore, while the items are not ordered, they are individually identified. Now if we regard ordering as simply one way of identifying items, it is possible that the extra demand imposed by ordering is similar to the demand imposed by identification. On the whole then it seems that, while digit span might not be an ideal measure with which to predict capacity to acquire any specific level, it probably is the most appropriate and practical measure available at present.

The decision to use digit span as a predictor of commutativity is broadly consistent with a number of other proposals in the recent literature to the effect that span is related to problem solving (Baddeley \& Hitch, 1974; Hitch, 1978; Hunt, 1978; Hunt, Frost \& Lunneborg, 1973; Keating \& Bobbitt, 1978). One qualification concerning this association should however be mentioned. Baddeley and Hitch (1974) used an interference technique to demonstrate the relationship between letter span and a simple reasoning problem. Interference with reasoning was found if participants were required to hold 6 letters in STM, but not if 3 were held. This may suggest that interference only occurs if the number of items in STM is near the maximum, and casts doubt on the proposition that STM and reasoning processes normally share a common storage space. However the reasoning task used by Baddeley and Hitch was quite simple, and amounted to requiring the participants to decide whether a statement about the order of occurrence of 2 letters was true or false. Interestingly, this is a level 1 reasoning task because it requires one relation (the order of the letters) to be represented internally and related to the stimulus. Such a task, according to our theory, would require 2 chunks for the representation to be applied to the problem. Since adult participants have a memory span of about 7, interference would not be expected if 3 items were already in STM, but it would be expected if 6 items were in STM. It appears then Baddeley and Hitch's data are consistent with our argument.

In assessing digit span norms, children are frequently assigned a score which relates to the longest series remembered correctly in a given number of trials. For instance, children may be given two series of each length, and given a score equal to the longest series which is recalled correctly in one trial. This procedure is used to guard against scores being depressed by distraction or through failure to perceive all the elements. Although it is a somewhat crude way of controlling for these extraneous factors, it is quite appropriate for our purposes, because we are only concerned with the maximum number of chunks which children can store, irrespective of whether they can do so on every trial. If a child requires $n$ chunks of information in store to check commutativity of a given cognitive system, then if she fails on 
one occasion, she may succeed on another. We are not concerned with predicting the specific trial on which success will be achieved, but only with whether it can be achieved.

We will now relate the information-storage demands of commutativity checking to STM norms. According to Method 1, a memory span of 2 would be required for level 1, 4 for level 2, and 6 for level 3. According to Stanford-Binet and Wechsler forms, average children acquire an STM capacity of 2 at age 2 years, 4 at age 4.5 years, and 6 at 10.5 years. These should therefore be the ages at which $50 \%$ of children can acquire the respective levels of systems. According to Method 2 on the other hand levels 1, 2, and 3 would require spans of 2, 3 , and 4, respectively. These levels should therefore be attainable at age 2, 3, and 4.5 in each case. Evidence from studies by Halford (1978) suggests that the ages of mastery correspond to those predicted by Method 1. This is corroborated by detailed study of protocols, which suggests that Method 1 does indeed correspond to the process which subjects actually employ.

We now have a set of predictions concerning the earliest age at which a given level of cognitive system can be attained. In a later section we will attempt to relate the system levels which have been defined to known cognitive developmental phenomena. If we can identify known performances which children can perform with cognitive system levels, we can predict the earliest age at which those performances can be acquired. If successful, this would permit us to explain cognitive developmental stages which have been identified empirically.

We are also able to derive a prediction about the nature of stages from the foregoing argument. This is that stage acquisition should be gradual, rather than sudden. This follows because the number of children with the information-processing capacity required for any given level of thought increases gradually with age; i.e., all children do not acquire memory spans of 4 immediately they reach the age of 4.5 years, but the number of children with a span of 4 increases gradually with age. Therefore we can use cumulative frequency curves for growth of memory span to predict the proportion of children in the population who should be capable of reaching any specific level. For instance, the curve for memory span of 6 shows that level 3 tasks should be learnable by $20 \%$ of 8 -year-olds, $35 \%$ of 10 -year-olds, $75 \%$ of 13 year-olds, etc.

This section completes the theoretical formulation of this paper. Our next step is to present empirical evidence concerning the validity of the theory. If the theory is found to be supported, we can then apply it to the task of explaining stage-related performances.

\section{EMPIRICAL EVIDENCE}

Both the experiments to be reported aim to externalize the process of constructing a cognitive system which is commutative. Basically this is done by requiring the child to arrange elements of the task in a way which is consistent with a previously learned system. A consistent arrangement corresponds to a commutative diagram, while an inconsistent arrangement does not.

Both studies are based on training which was carried out in three main phases, designed to make gradually increasing cognitive demands. The first phase, called symbol system training (SST), is designed to simply teach the task system. The second phase, called operator application training $(O A T)$, is designed to teach participants to use level 1 cognitive systems, and to ease the transition to the final phase. The third phase, called cognitive system training (CST), is criterial, and is designed to manipulate the level of cognitive system which the child is required to employ, in order to study the interaction of this factor with age. 


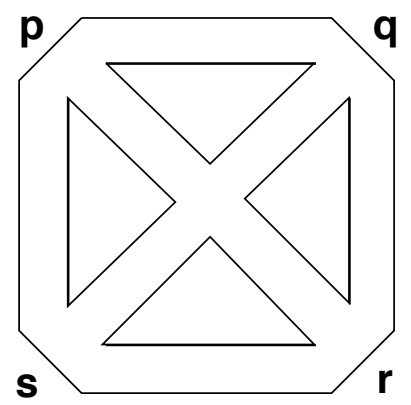

FiguRE 17. Environment used in Experiment 1. The letters are shown for convenience in exposition, but the corners were unmarked on the apparatus. The sides of the square measured $35.5 \mathrm{~cm}$.

TABLE 1

Task Systems Used in Experiment 1

\begin{tabular}{cccccccc}
\hline & System A & & \multicolumn{5}{c}{ System B } \\
\hline & \multicolumn{3}{c}{ Operators } & & \multicolumn{3}{c}{ Operators } \\
\hline States & $\mathrm{N}$ & $\mathrm{C}$ & $\mathrm{A}$ & States & $\mathrm{N}$ & $\mathrm{C}$ & $\mathrm{D}$ \\
\hline $\mathrm{p}$ & $\mathrm{p}$ & $\mathrm{q}$ & $\mathrm{s}$ & $\mathrm{p}$ & $\mathrm{p}$ & $\mathrm{q}$ & $\mathrm{r}$ \\
$\mathrm{q}$ & $\mathrm{q}$ & $\mathrm{r}$ & $\mathrm{p}$ & $\mathrm{q}$ & $\mathrm{q}$ & $\mathrm{r}$ & $\mathrm{s}$ \\
$\mathrm{r}$ & $\mathrm{r}$ & $\mathrm{s}$ & $\mathrm{q}$ & $\mathrm{r}$ & $\mathrm{r}$ & $\mathrm{s}$ & $\mathrm{p}$ \\
$\mathrm{s}$ & $\mathrm{s}$ & $\mathrm{p}$ & $\mathrm{r}$ & $\mathrm{s}$ & $\mathrm{s}$ & $\mathrm{p}$ & $\mathrm{q}$ \\
\hline
\end{tabular}

The logic of the experiment need only be described for Experiment 1, since it is basically the same for both experiments. Experiment 1 utilized a cardboard square as shown in Fig. 17, and children were taught to move a toy truck around the square in accordance with one of the systems shown in Table 1. Each system consists of states $p, q, r, s$, and three of the four operators, $N, C, A, D$. In the first two phases of training ( $S S T$ and $O A T$ ), the states were identified with the corners of the square as shown in Fig. 17 so that operator $N$ was null and meant to remain in the same place; $C$ meant a clockwise movement from $p$ to $q, q$ to $r$, etc., $A$ meant an anticlockwise movement from $p$ to $s, \mathrm{~s}$ to $r$, etc., and $D$ meant a diagonal movement from $p$ to $r, r$ to $p$ etc. Each operator was represented by a geometric figure (triangle, circle, dumbbell, etc.). A white cardboard house was placed at each corner of the square, and a toy truck was placed in front of one of the houses. The child was shown a cue card containing a geometric figure representing one of the operators, and had to learn by trial and error to move the toy truck in accordance with the operator represented by the card. For instance, if triangle represented operator $\mathrm{D}$, and if the house was placed at $\mathrm{q}$, then the correct response is to move the truck to s. Learning of one problem was complete when the child could consistently interpret all cue cards in accordance with the operators they represented. Three new cue cards were then randomly allocated to the operators, and training on a new problem was carried out in the same way. Thus a learning set procedure was used to teach the child a concrete embodiment of the system. The aim was to insure that the child stored the system in longterm memory. That is, the child should have learned the four states and the way in which each operator effects a change from one state to another. At the conclusion of this training, the child should have a symbol system containing information equivalent to system $A$ or system $B$ (depending on which one was used for that child) in Table 1.

The criterial phase of the task $(M)$ required the child to relate the symbol system to a problem with the same structure as those previously learned, but with new elements. Each 
problem began with three unknown cue cards, and the states were represented by different coloured houses, only one of which was placed on the board by the experimenter. However the four colours were in fact randomly allocated to the states in a different way for each problem, although this allocation was known only to the experimenter when each problem began. A sample problem is shown in Table 2.

In this phase the operators were defined as changes from one colour to another. This meant that the child was required to learn, and was given feedback about, movements from one colour to another. For example, in Table 2 the child would have to learn that cross meant to go from red to green, green to blue, etc. It was up to the child to place the houses (except the first one) around the square in an appropriate way. Furthermore, he was given no feedback for the way he did this; the only feedback was for saying that a cue card meant to go from a house of one colour, to a house of' another colour. The sole criterion of a correct arrangement of houses around the square therefore is consistency with the system.

TABLE 2

Example of Problem Based on System B in Table 1

\begin{tabular}{cccc}
\hline & \multicolumn{3}{c}{ Cue cards } \\
\cline { 2 - 4 } House colours & Circle & Cross & Star \\
\hline Red & Red & Green & Blue \\
Green & Green & Blue & Yellow \\
Blue & Blue & Yellow & Red \\
Yellow & Yellow & Red & Green \\
\hline
\end{tabular}

Of the 24 possible arrangements of houses around the square, 8 are consistent with the system. To consider the example problem in Table 2, it would be consistent with the system if red was placed at $p$, green at $q$, blue at $r$, and yellow at $s$. (The reader will probably find it helpful in following this argument if he/she draws a sketch of the square, and writes in the house colours as the argument proceeds.) With such an arrangement, cross will mean to go one step clockwise, star one step anticlockwise, and so on. Another consistent arrangement would be if all houses were moved one step clockwise; i.e., with red at $q$, green at $r$, etc. Alternatively, the houses could be arranged anticlockwise, with red at $p$, green at $s$, blue at $r$, and yellow at $p$. Cross would now mean to go one step anticlockwise, etc.

For an example of an inconsistent arrangement, consider placing red at $p$, blue at $q$, green at $r$, yellow at $s$. Cross now means to go diagonally from red to green and anticlockwise from green to blue; i.e., it has no consistent meaning in terms of the previously learned operators.

If a consistent arrangement is chosen, it is easy to predict the correct house colour for any operator, and the task would therefore be easy to learn. With an incorrect arrangement, prediction and learning are difficult. Therefore, although the child is given no feedback about the arrangements as such, the requirement of learning to predict the house colour which results from any operator provides a strong incentive to make a consistent arrangement. Children were permitted to rearrange the houses as often as they wished in order to find one which was consistent.

Now we will show how this task requires the child to construct a cognitive system which commutes. The symbol system which the child must use is the previously learned system $A$ or system $B$ as shown in Table 1. (The system would probably be stored in memory as images of movements clockwise, diagonally, etc., or as verbal rules like - "... go this way ..." but our concern here is only with the fact that the child has stored in memory a system equivalent to 
one of those in Table 2) The environment system is the set of elements used in any specific task, together with the relations between these elements. Thus, in the example we have been using, the environment system is summarized in Table 2 . Now we will show how a consistent arrangement corresponds to a diagram which commutes.

The consistent arrangement mentioned earlier corresponds to the following set of mappings.

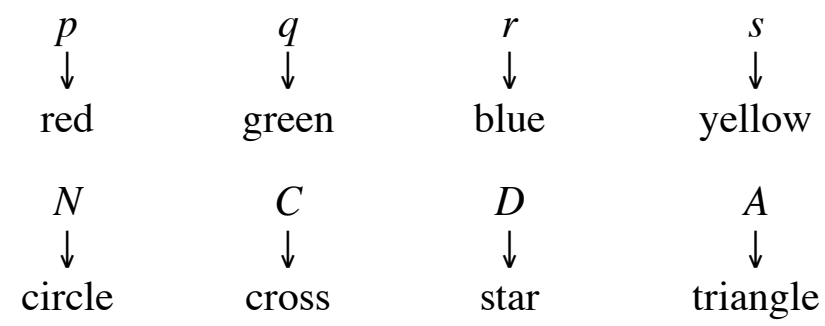

Now we consider operator $C$ applied to $q$, and the resulting commutative diagram is shown in Fig. 18. Going along the top line, $C$ applied to $q$ produces $r$, and a second application of $C$ produces $s$ (Table 1). Going along the bottom line, one application of cross to green produces blue, and a second application produces yellow (Table 2). Since s is interpreted as yellow, both paths yield the same answer, or the diagram is commutative.

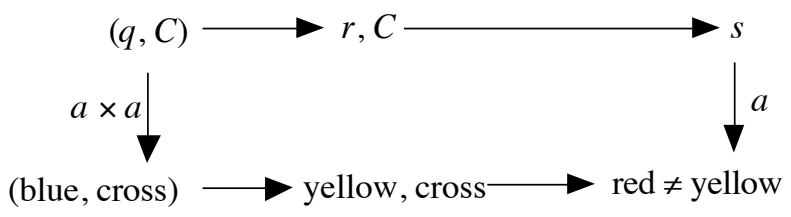

FIGURE 18

The inconsistent arrangement mentioned earlier can be represented as the following set of mappings.

$\begin{array}{cccc}p & q & r & s \\ \downarrow & \downarrow & \downarrow & \downarrow \\ \text { red } & \text { blue } & \text { green } & \text { yellow } \\ & & & \\ N & C & D & A \\ \downarrow & \downarrow & \downarrow & \downarrow \\ \text { circle } & \text { cross } & \text { star } & \text { triangle }\end{array}$

For the example of operator $C$ applied to $q$, the commutative diagram would be as shown in Fig. 19. Going along the top line, one application of $C$ to $q$ produces $r$, and a second application produces $s$. Going along the bottom line, one application of cross to blue produces yellow, and a second produces red (Table 2). However since $s$ is not mapped into red, the interpretation is inconsistent and the diagram is not commutative.

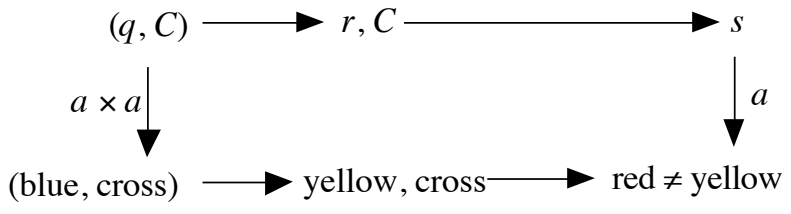

FIGURE 19 
Now we can express these commutative diagrams, in general form. First, we will define the environment for the sample task (Table 2), consisting of state elements $\left(E_{s}\right)$ and operator elements $\left(E_{o}\right): E_{s}=$ red, green blue, yellow, $E_{o}=$ circle, cross, star, triangle.

Secondly, we define the symbol elements, comprising states $\left(S_{s}\right)$ and operators $\left(S_{o}\right): S_{s}=$ $p, q, r, s, S_{o}=N, C, D, A$.

Table 1 expresses all the information in the symbol system, while the environment contingencies corresponding to one specific problem are expressed by Table 2 .

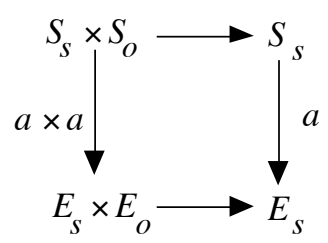

FIGURE 20

If the interpretations of $S_{s}$ as $E_{s}$ and $S_{o}$ as $E_{o}$ are consistent, the diagram Fig. 20 would be commutative. It is possible within this paradigm to vary the system level while holding the procedure constant. In fact system $A$ in Table 1 is a level 1 task and system $B$ is a level 2 task. The reason for this is that in system $B$ the operators must be interpreted in pairs in order to decide whether an arrangement is consistent or not. The reader will probably have noticed that in the commutative diagrams in Figs. 18 and 19 the operators were interpreted in pairs (i.e., two occurrences of $C$ are used in both cases) and the reason is that the diagrams were based on the assumption that $C$ came from system $B$, and two occurrences of the operator are needed to check commutativity in this system.

An example will make this clear: suppose there is a red house at position $q$, a cue card containing a cross is shown, and the correct response is a green house (red, cross $\rightarrow$ green). If green is placed at $r$, this is tantamount to interpreting cross as $C$. If green is placed at $s$, cross is interpreted as $D$. It is impossible to tell which interpretation is correct. They can however be distinguished on the basis of two trials jointly; e.g., red, cross $\rightarrow$ green; green, cross $\rightarrow$ yellow jointly imply that cross must be interpreted as $C$. Cross cannot be $D$ because this interpretation would imply a return to the starting point. Notice also that operators only are interpreted because, since states and operators are defined relative to one another, it is only necessary to interpret one or the other, but not both. Thus if both cross cues are interpreted, this implies an interpretation of the colours which will be consistent with the system. Alternatively we could have interpreted the colours, with the operator interpretations being implied. The former is chosen simply because it is more consistent with subject protocols. However it is true that, whichever style of interpretation is chosen, task elements have to be interpreted in pairs. This is what makes the system level 2.

The reason why system $A$ does not impose this requirement, and therefore is not level 2 , is that operators $A$ and $C$ are interchangeable. This is in fact what enables the informationprocessing requirements of the system $A$ task to be reduced while holding the number of items constant. The result is that there is no sense in which it is an error to interpret a cue card as $A$ when it was designed to be $C$ or vice versa, since both are consistent with the system. A single trial is sufficient to determine whether a cue represents the null or one of the non-null operators (if it means to go from one colour to another, it must be the latter). A house placement in terms of either a $C$ or an $A$ operator will then be sufficient to insure consistency with the system. 
It is interesting to note that hypotheses about the meanings of cue cards in level 2 tasks cannot be tested by interpreting less elements than the level of system requires; i.e., since a level 2 system has its elements defined in pairs, hypothesis testing must proceed on the basis of pairs of elements. Let us consider what might happen if an attempt were made to circumvent this requirement, as for instance if participants tried to interpret one element at a time: given an item such as red, star $\rightarrow$ blue, where star really represents $D$, the child who hypothesizes that star represents operator $C$ might place red at $p$ and blue at $q$. He might then recognize that movement from $q$ to $p$ on the second occurrence of star is inconsistent with rule $C$, since he interprets $C$ as a clockwise movement. This could be overcome by reversing the houses, but the first movement would then be wrong. Thus the attempt to interpret the task elements one at a time only leads to further inconsistencies.

Clearly therefore the level 2 CST task requires elements to be interpreted in pairs. This follows in a general sense from the theory, and is illustrated for a specific case in the example above. It implies that two symbol elements would have to be matched to two task elements in order to make an interpretation. The information load therefore is four chunks: two symbol elements and two task elements. This is in accord with line 2 of the Method 1 calculation of the information load for level 2 tasks in an earlier section. On the other hand, it is possible that, in accordance with Method 2, the peak information load could be reduced to three chunks by first combining the two operators into the single operator to which they are equivalent (cf. the Method 2 calculation for level 2, line 2). The information processing load for the level 1 task peaks at two chunks, whichever method is used. Either way therefore the minimum age of attainment should be higher for level 2 than for level 1. It is proposed to test this prediction in an experiment using two levels of age and two levels of task. If the ages are appropriately chosen, so as to span the transition point for level 2 capability, we should obtain an age by task levels interaction with a specific form: both age groups should succeed on level 1 , but only the older age group should succeed on level 2.

\section{EXPERIMENT 1}

\section{Method}

Participants. There were 40 preschool children ages 4.0 to 4.11 (mean age $=4.6$ ) and 40 Grade 1 children ages 5.5 to 6.5 (mean age $=6.0$ ). Both the preschool and the primary (elementary) school were in a middle-class socioeconomic suburb of Brisbane, Australia. The schools were physically close together and drew their pupils from the same section of the community. Half of each sample was allocated to the system $A$ (level 1) condition, and half to the system $B$ (level 2) condition at random.

Procedure. Subjects were tested individually in from 9 to 14 sessions, whose length varied from 5 to $15 \mathrm{~min}$, depending on subject interest. The experiment consisted of four phases: symbol system training (SST), operator application training $(O A T)$, cognitive system training (CST), and a CST transfer test. Each child was taught either system $A$ or system $B$ throughout.

$S S T$ began by placing four white cardboard houses on the corners of the square. The toy truck was placed in front of one of the houses selected at random, and the child was shown a cue card which represented one of the operators. She/he then moved the truck according to what she/he thought the cue card meant. If this was incorrect, she/he was so informed and invited to try again, and so on until the correct movement was found.

The training procedure included several features designed to maximize learning:

(1) The movements corresponding to each operator were carefully explained and demonstrated before training began.

(2) The child was asked to imitate each movement. 
(3) At the end of each trial the child was taught to verbalize the movement (clockwise, anticlockwise, diagonal, etc.) which was correct for each eve card, or to indicate it by gesture.

(4) Participants were encouraged to name the cue cards to enhance discriminability.

(5) Only one step ( $p$ to $q, q$ to $r$, etc.) was required on each trial, but the trials were organized into sets which made the nature of the operator clear. Thus a sequence using operator $C$ might comprise four trials with movements from $p$ to $q, q$ to $r, r$ to $s$, and $s$ to $p$.

Training continued on the single SST problem for 144 trials, or until 12 error-free trials occurred. Three $O A T$ problems were then given.

Each $O A T$ problem began with an $S S T$ problem, which was learned to the same criterion as before. The cue cards used in the SST phase of the problem were retained, but the white houses were replaced by the coloured houses, which were not placed on the board prior to the beginning of the problem. The operators were now defined as movements from one colour to another, so the child had to learn to specify the correct colour of house to which the truck should go in response to each cue card. The child then had to place the houses on the board in a way which was consistent with the previous meaning of the cue cards.

To see how this works, suppose that the green house had been placed at $p$ on the square, and the cue card representing operator $C$ had been defined as a movement from the green house to the blue house, from blue to red, red to yellow, and yellow to green. Then with the truck at green, the correct response to this cue card would be blue, and the blue house should be placed at $q$. Similarly, the red house should be placed at $r$ and yellow at $s$. A different random allocation of houses to states in the system was made for each problem.

If the subject's placement was incorrect, the experimenter said: "is that the way this message (card) told the truck to go when we had the white house". The subject was then asked to "... fix it up so that this message will be like it was when we had the white houses" (prompting procedure). If the child still did not succeed in making correct arrangement, the experimenter demonstrated the necessary shifts and explained how the houses were then arranged to that the messages meant the same as before (demonstration procedure).

On the first $O A T$ problem the placements were corrected item by item, but on subsequent problems correction was made after all four houses had been placed. In the event that the child had managed to arrange the houses correctly without any assistance, the experimenter placed them incorrectly at the conclusion of the problem, and then used the procedure above to illustrate how such an incorrect arrangement would have been corrected. This was to insure that all participants knew how to correct inappropriate arrangements.

Notice that in $O A T$ the states are still, in effect, defined as locations because there is only one arrangement of the houses which is consistent with the immediately preceding SST problem. This is because the cue cards have already been learned as specific movements; clockwise, diagonal, etc.

Following OAT three problems of $C S T$ were given. $C S T$ was similar to $O A T$ except that the subjects did not have the opportunity to learn the meaning of the cue cards beforehand. Each problem began with three unknown cue cards, and with the four coloured houses randomly allocated to the states, $p, q, r, s$. The procedure began by placing a randomly selected house at the appropriate point on the square. The child was given the other three houses to hold. A cue card was shown, and the child was asked to choose the house to which the truck should go. When the correct house was chosen (sometimes after trial-and-error), the child was told it was correct and was then asked to place it on whatever corner of the square he thought was appropriate. The truck was then placed in front of that house, another cue card was presented, and the procedure repeated for the next trial. No feedback was given for the placements, but it was emphasized that the subject was free to rearrange the houses whenever he wished, and that the houses should be arranged so that the cue cards would mean the same as the messages 
in all the previous games. Each CST problem was continued for 72 trials or until 12 consecutive error-free trials occurred.

A correct response was defined as a correct prediction of house colour. Suppose for instance that, in a particular problem, the green house was in position at location $q$, a cross cue was shown, and the correct response (from Table 2) was for the truck to go to the blue house (green, cross $\rightarrow$ blue). The child is credited with a correct response if the correct colour, blue, is predicted for the combination of green with cross. The child is credited with succeeding on a given problem if 12 consecutive correct predictions are made.

Since participants were encouraged to rearrange the houses, there is clearly a possibility of a correct arrangement being made by chance, and the probability of this happening increases with the number of rearrangements made. However, it is essential both to encourage rearrangement and to use a large number of trials to maximize the chance of reaching criterion in order to insure that even the youngest participants had ample opportunity to learn. Nevertheless, provided we only use the CST task to examine the age by task levels interaction, the spuriously high success rate will not affect the validity of the experiment. In fact, since previous work (Halford, 1978) has shown more rearrangements in the system $B$ condition, owing to the greater difficulty of finding a consistent arrangement in that task, then a spuriously high performance will be most likely in that condition. It will also be higher for 4year-olds because they will have greater difficulty finding a correct application of the system to the task. Therefore this factor works against finding a significant interaction, and consequently works against the authors' hypothesis.

Since the absolute level of performance on the system $B$ task is also of interest, a transfer test is used to assess it. Ten trials are used, which means that the information necessary to correctly arrange the houses is provided four times to each subject. From previous work it is known that one or two rearrangements will be made in this time by most children, with the result that it is unlikely that a correct arrangement will be made by simply shifting houses around until one is stumbled upon. A chance correct initial arrangement is also eliminated by making the first house placed incorrect. For instance, if a problem begins with an item such as red, triangle $\rightarrow$ blue, with red at $p$, then if the child places blue at $r$ (effectively interpreting triangle as operator $D$ ), triangle will be made to represent operator $C$, so that blue will have to be shifted to either $q$ or $s$.

In the system $B$ condition this does not conflict with anything the child has learned, because this condition is designed to require a level 2 cognitive system, with the result that there is insufficient information in one trial to determine the correct interpretation of a cue. In the system $A$ condition one trial does provide sufficient information to place a house owing to the fact that the system A CST task is designed to require only a level 1 cognitive system. Therefore it would conflict with previous information if a house placed in accordance with the first trial was subsequently found to be incorrect.

Accordingly, three 10-trial transfer problems were given in the system $B$ condition following CST. The first placement was made incorrect as described above. In other respects the procedure was the same as for CST. The sole criterion of success was whether a correct arrangement was made.

Each participant was given a digit span test designed to separate subjects with digit spans of less than 4 from those with spans of 4 or more, making maximum allowance for errors due to distraction. This was done by crediting subjects with having passed the longest series which they correctly recalled on three or more trials out of six. Error types and frequencies had been determined in a previous unpublished study using 93 subjects and it was found that the chance of obtaining three out of six correct by guessing $\approx 0.08$. Therefore this criterion allows participants up to three errors due to distraction, while requiring them to perform better than 
chance at an acceptable level of confidence. Re-test reliability, also using the earlier sample of 93 subjects, was found to be 0.73 after 3 months.

A tape-recorded, ungrouped, monotone presentation was used at the rate of one digit per second. Each participant was given six series of each length, starting at length three. If three out of six were passed, the next longest set was given, until a set was failed.

\section{Predictions}

The cognitive systems which are required at each phase of the task are summarized in Table 3. No cognitive system is required in SST because all the information required for the interpretation of the task elements is provided by the procedure, and the participant does not have to use an internal representation of the system to interpret them. The houses are already in position, and full feedback is provided concerning the meaning of the operators. In $O A T$ the houses must be arranged in a way which is consistent with cue cards which represent known unary operators, so level 1 systems are required. In $C S T$ system $B$ requires a level 2 cognitive system and system A a level 1 cognitive system as explained earlier.

The predictions from the theory would be that there will be no age difference in success rate in SST or $O A T$ because these require either no cognitive system or a system which is within the capacity of participants in both age groups. The same would be true for the system A CST task. There should however be age-related differences in success rate in the system $B$ CST task, because this requires a level 2 cognitive system which should be beyond the capacity of 4-year-old children. Thus the age by task levels interaction predicted by the theory should be observed in the CST task.

TABLE 3

Level of Cognitive System Required in Each Phase of Experiment 1, for Each Task System

\begin{tabular}{|c|c|c|c|c|}
\hline \multicolumn{5}{|c|}{ Phases } \\
\hline & $S S T$ & $O A T$ & $C S T$ & Transfer \\
\hline System $A$ & $\begin{array}{l}\text { No cognitive } \\
\text { system required }\end{array}$ & $\begin{array}{l}\text { Level } 1 \text { cognitive } \\
\text { system required }\end{array}$ & $\begin{array}{l}\text { Level } 1 \text { cognitive } \\
\text { system required }\end{array}$ & $\begin{array}{l}\text { No transfer } \\
\text { task possible }\end{array}$ \\
\hline System $B$ & $\begin{array}{l}\text { No cognitive } \\
\text { system required }\end{array}$ & $\begin{array}{l}\text { Level } 1 \text { cognitive } \\
\text { system required }\end{array}$ & $\begin{array}{l}\text { Level } 2 \text { cognitive } \\
\text { system required }\end{array}$ & $\begin{array}{l}\text { Level } 2 \text { cognitive } \\
\text { system required }\end{array}$ \\
\hline
\end{tabular}

If there are any 4-year-olds who succeed on the system $B C S T$ task by making chancecorrect arrangements, they should not succeed on the transfer task, since the transfer task procedure would make chance successes very unlikely. Accordingly the transfer task should give a reliable indicator of the absolute levels of success which are possible for 4-year-olds.

These predictions have been based on the assumption that consistency checking by subjects will be carried out by a process which is equivalent to Method 1. This is because previous work (Halford, 1978) revealed protocols which were more consistent with Method 1 than Method 2, and this was supported by the approximate age norm obtained. If this assumption is incorrect, we should find that 4-year-olds will master level 2 tasks, since their informationprocessing capacity should be equal to that which would be required for Method 2 calculations at level 2. It would then be appropriate to carry out a further experiment contrasting 2- and 3-year-olds on level 1 and level 2 tasks. 


\section{Results}

Each participant was given one point for each CST problem on which criterion was reached, and these scores were subjected to analysis of variance. This yielded a significant effect of age, $F(1,76)=7.56, p<0.01$, and of task levels, $F(1,76)=19.72$. There was also a significant age by task levels interaction, $F(1,76)=5.41, p<.025$. The number of $C S T$ problems on which criterion was reached is shown in Table 4. The correlation between reaching criterion and making a correct arrangement of the houses was 0.83 .

TABLE 4

\begin{tabular}{lcc}
\hline \multicolumn{3}{c}{ Number of Cognitive System Training Problems on Which Criterion Was Reached } \\
\hline & System A & System B \\
4-year-olds & 2.95 & 2.15 \\
5- to 6-year-olds & 3.00 & 2.75 \\
\hline
\end{tabular}

These data show that the main prediction of the theory with respect to age by levels interaction is confirmed. The transfer data, as shown in Table 5, again show the 5- to 6-yearolds to be better than the 4-year-olds $\left(\chi^{2}(1)=13.74, p<0.001\right)$. We also see that, if we define success as two correct arrangements out of three, only four subjects, or $20 \%$ of the 4-year-olds mastered this task. This result was not due to the 4-year-olds making insufficient attempts to arrange the houses. In fact the 4-year-olds made a total of 89 attempts, of which 17 were correct, whereas the 5- to 6-year-olds made 80 attempts of which 43 were correct.

TABLE 5

Frequency with Which Correct Arrangements Were Made on System B Transfer Task

\begin{tabular}{lllll}
\hline & \multicolumn{5}{c}{ Number of correct arrangements } \\
\cline { 2 - 5 } & 0 & 1 & 2 & 3 \\
\hline 4-year-olds & 8 & 8 & 3 & 1 \\
5- to 6-year-olds & 2 & 3 & 5 & 10 \\
\hline
\end{tabular}

Although the above analysis is all that is required to test the predictions of the theory, for the sake of completeness, and to give more insight into the overall behaviour of the children on this (relatively novel) task, performance data from the other phases should also be reported. Since all participants reached criterion on all SST and OAT tasks, error and trials to criterion data will be analysed.

In $S S T$ there was a significant effect of age in errors, $F(1,76)=28.02, p<0.001$, and in trials, $F(1,76)=36.15, p<.001$. In errors the preschool mean was 25.78 and the Grade 1 mean was 11.43 , while the corresponding means in trials were 84.60 and 48.00 . Task level and the interaction of task level with age were both nonsignificant in SST. These results reflect simply more rapid learning by the older subjects.

In $O A T$ there was a significant effect of age in errors, $F(1,76)=5.76, p<0.02$, and in trials, $F(1,76)=8.61, p<0.005$. In errors the preschool mean was 2.53 and the Grade 1 mean was 1.97 , while for trials the means were 25.44 and 23.16 , respectively. These scores reflect merely the time taken to arrange the coloured houses in relation to the rules which were already known, hence the small number of both errors and trials. Task was significant in 
trials, $F(1,76)=4.71, p<0.05$, but not in errors. Trial means were 25.08 for system $A$ and 23.40 for system $B$. The interaction between age and task was significant in errors, $F(1,76)=$ 7.61 , but not in trials. The error means were as follows: for preschool subjects, system $A$ was 2.23 and system $B$ was 2.80 ; for Grade 1 subjects the system $A$ mean was 2.35 and system $B$ was 1.60 . The effect of problems was not significant. Again, the only consistently reliable differences in $O A T$ reflect simply age differences in learning rate.

On the first $O A T$ problem it was not possible to assess the children's ability to arrange the houses, because the amount of help given by the experimenter was too large in some cases. However, on Problems 2 and 3, all subjects were able to find the correct arrangement without the need for the experimenter to demonstrate. Thirty-three of the eighty subjects needed prompting to draw their attention to the fact that their initial arrangement was incorrect on both problems, and nine needed prompting on one problem. There was no significant association between either task level or age and the number of prompts needed.

The digit span scores of four of the 5- to 6-year-olds could not be used because of an experimental error. The data for the remaining subjects showed that only four of the twenty 4year-olds and all sixteen of the remaining 5- to 6-year-olds had spans of four or more. However, all but one of the 4-year-olds had spans of three or more. The first conclusion then is that capacity to learn level 2 systems requires a span of four rather than a span of three, since the majority of the 4-year-olds did not succeed on the level 2 task. Second, since the majority of the 5- to 6-year-olds had spans of no more than four and mastered the level 2 task, it seems that a span of four predicts the age mastery of level 2 systems quite well. It seems reasonable to assume that most or all of the remaining 5- to 6-year-olds would also have had spans of four or better, since the ability of the sample was clearly above average.

Of the 16 subjects with digit spans of less than four, 2 achieved the criterion of two or more problems passed in the transfer test. Of the 20 subjects with spans of four or more, 14 reached this criterion. This difference is significant $\chi^{2}(1)=11.90, p<0.01$. This relationship also corresponds to the 4) coefficient of 0.57 . It would be interesting to carry out further correlational analysis, but since the age distribution is bimodal and is not continuous, the risk of spurious results is too great.

\section{Discussion}

The results of the experiment show an interaction which has a form consistent with our predictions as stated earlier; i.e., both age groups readily attain level 1 tasks, but the level 2 tasks are much harder for the 4-year-olds than for 5- and 6-year-olds. The 4-year-olds mostly had digit spans of less than four chunks, whereas the 5- and 6-year-olds had spans of four chunks or more, so the interaction has a form consistent with that predicted by applying Method 1 to the problem of calculating the information-processing capacity required for a commutative cognitive system.

On the other hand, it is true that an appreciable number of 4-year-olds reached criterion on level 2 CST tasks, but this was anticipated because it is possible to find a correct arrangement of houses around the square by trial-and-error. Allowance was made for this by including a transfer task which was designed to avoid correct arrangements occurring by trial-and-error. On this task, as shown in Table 5, only four out of twenty 4-year-olds reached the criterion of finding a correct arrangement on two out of three problems. This proportion is consistent with the proportion who had digit spans of four. Therefore the absolute level of performance on the level 2 task by 4-year-olds is also consistent with the theory.

The lower level of performance of the 4-year-olds on the level 2 CST task is made more significant by the fact that two lines of evidence show that they can learn level 1 tasks. Thus they perform perfectly on the CST task using system $A$, showing that it is not the CST 
procedure which is responsible for the deficit with system $B$ in the CST task. They also perform adequately in the $O A T$ procedure with system $B$, showing that it is not the individual elements of the system which are responsible. It seems clear therefore that the only interpretation which fits the total pattern of the data is that 4-year-olds can use level 1 but not level 2 cognitive systems.

Detailed examination of protocols indicated that most participants performed the CST task by hypothesizing a specific movement to be performed in response to each cue card. The houses would be placed on the board in a way which was appropriate to this movement, and the hypothesis would be changed following disconfirming evidence. This hypothesis testing procedure is equivalent to calculation Method 1. That is, testing hypotheses means interpreting the elements, then calculation the result and testing for consistency.

\section{EXPERIMENT 2}

Experiment 2 sought to determine the age at which children could learn level 3 cognitive systems. The design was similar to that used in Experiment 1, with two levels of age and two levels of system (level 2 and level 3).

\section{Method}

Apparatus. The apparatus included a set of paths forming a hexagon on a white cardboard square as shown in Fig. 21. The pathways which corresponded to each of the operators were coloured to facilitate discrimination between different operators. There were also six identical white cardboard houses, and one house of each colour red, brown, pink, purple, gold, and orange. The white square measured $51 \mathrm{~cm}$ to a side. The houses, toy truck, and cue cards were similar to those used in Experiment 1 .

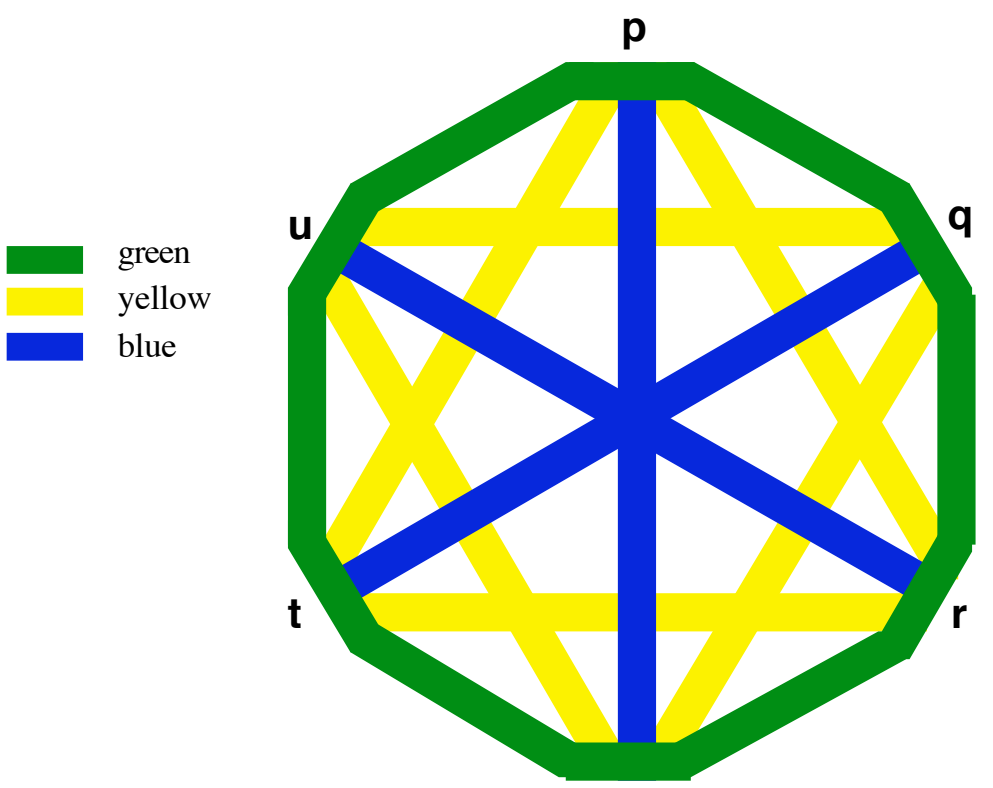

FIGURE. 21. Hexagonal array used in Experiment 2. The letters are shown for convenience in exposition, but the corners were unmarked on the apparatus.

Subjects. There were 20 primary (equivalent to USA elementary) school children ages 8.0 to 9.5 (sample mean $=8.10$ ) and 20 ages 9.6 to 10.11 (sample mean $=10.4)$, from a middle class area of Brisbane, Australia. The mean I.Q. of the sample, based on either the ACER Junior Nonverbal test or the ACER revision of the Jenkins Nonverbal test, was 120 . The approximate mean mental age of the younger sample would therefore be 10.6 and for the older sample would be 12.4.

Systems used. The systems used in Table 6 were employed. The level of cognitive system required at each phase for system $C$ and system $D$ is shown in Table 7 . 
The explanation of cognitive system levels required in SST and $O A T$ is the same as in Experiment 1. However, in the system $C$ condition $C S T$ requires that operator 1 be distinguished from operator 3 , which can be done by considering two items jointly. For instance, if a cue card means to go from red to brown and brown to red it must represent operator 3 , because this is the only operator which matches these two actions. The symbol system required then is essentially the same as for the level 2 task in Experiment 1.

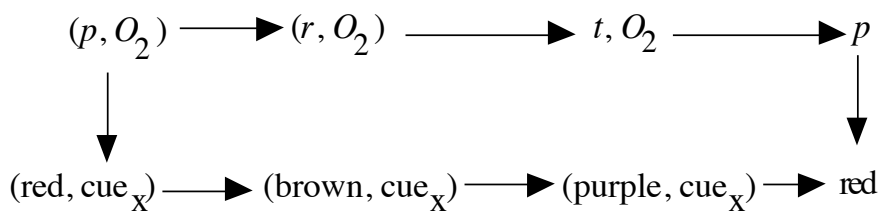

This can be recoded as:

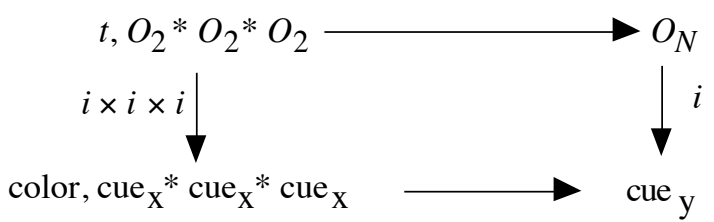

FIGURE 22

In the system $D$ condition however operator 2 must be distinguished from operator 1 , and this can only be done by considering three items jointly. For instance, if a cue card means to go from red to brown, brown to purple, and purple to red it represents operator 2, whereas if it means to go from red to brown, brown to purple, and purple to gold it represents operator 1. Matching a composition of three operators to three cue cards requires a symbol system which is equivalent to a ternary operation. The cognitive system required could be represented by diagrams in Fig. 22.

Procedure. The procedure was basically the same as in Experiment 1. Each participant was given two problems of SST, two problems of $O A T$, and five problems of CST. A transfer task was unnecessary because there are 720 possible arrangements in this task of which only 12 are correct. There is therefore only a very small probability of obtaining a correct arrangement by chance. The number of sessions required varied from 5 to 10 , and the length of sessions varied from 15 to $30 \mathrm{~min}$.

TABLE 7

Level of Cognitive System Required in Each Phase of Experiment 2, for Each Task System

\begin{tabular}{lccc}
\hline & \multicolumn{3}{c}{ Phases } \\
\hline System C & No cognitive & OAT & CST \\
system required & $\begin{array}{c}\text { Level 1 cognitive } \\
\text { system required }\end{array}$ & $\begin{array}{c}\text { Level 2 cognitive } \\
\text { system required }\end{array}$ \\
Nystem D & $\begin{array}{c}\text { No cognitive } \\
\text { system required }\end{array}$ & $\begin{array}{c}\text { Level 1 cognitive } \\
\text { system required }\end{array}$ & $\begin{array}{c}\text { Level 3 cognitive } \\
\text { system required }\end{array}$ \\
\hline
\end{tabular}

On the first SST problem, participants were trained for six blocks of 16 trials or until criterion of 10 error-free trials occurred, and on the second SST problem they were trained for a maximum of four blocks of 16 trials. In $O A T$ four blocks of trials were used, and six blocks in CST.

In this experiment it is possible to use the procedure of making participants' initial house placements wrong in $C S T$ problems with the $C$ and $D$ systems. For example, suppose that a problem began with a red house at $p$, and a triangle cue card which meant to go from red to brown. If the child placed the brown house at $q$, he/she would in effect by hypothesizing that triangle represented rule 1 . For that problem then, triangle would be made to represent rule 2 or 3 , depending on the condition to which the participant was assigned. 


\section{Results}

Participants were given one point for each CST problem on which criterion was reached. Both age groups obtained perfect scores in the system $C$ condition, but in the system $D$ condition the mean number of successful problem attempts was 2.70 for the 8.0-9.5 age group and 4.60 for the 9.6-10.11 age group. Analysis of variance of these scores showed a significant effect of age, $F(1,36)=7.65, p<0.01$, a significant effect of level, $F(1,36)=$ $15.44, p<0.001$, and a significant interaction, $F(1,36)=7.56, \mathrm{p}<0.01$. There was a perfect correlation between reaching criterion and making a correct arrangement.

An analysis of the other phases was carried out for the sake of completeness. In SST the only significant effect was for problems; $F(2,72)=3.68, p<0.05$, in errors, but this effect was not significant for trials. The mean errors were: Problem 1, 1.58; Problem 2, 1.03; Problem 3, .83 . The $S S T$ phase of the first $O A T$ problem is able to be included in this analysis because it is effectively continuous with the SST procedure. In $O A T$ there was a significant effect of problem number on trials, $F(1,36)=7.50, p<.01$, but not on errors. The mean trials were: Problem 1, 15.3; Problem 2, 14.08. The effect of age and level were nonsignificant for both errors and trials. Thus there were no consistently reliable effects in the first two phases. All participants reached criterion on all SST and OAT problems.

Seven of twenty 8.0-to-9.5-year-old children had digit spans of six or more, whereas fifteen of twenty 9.6-to-10. 11-year-old children had spans of six or greater. This difference is significant $\chi^{2}(1)=6.46, p<0.02$. The biserial correlation between digit span of six or more versus a span of less than six and number of CST problems on which criterion was reached is $0.74, d f=18, p<0.01$. When age is partialled out this becomes $0.64, d f=17, p<0.01$.

An attempt was made to probe further the subjects' strategies on the level 3 task, in order to obtain information relevant to the question of why failures occurred at this level. The appropriateness of subjects' attempts to rearrange the houses when they changed hypothesis was analysed. An arrangement was considered to be appropriate if, on changing hypothesis, the subject rearranged all relevant houses in a way which was consistent with the three preceding trials which provided relevant information. In most cases this was in fact the three immediately preceding trials, but it occasionally happened that there was one uninformative trial in the last three, in which case this trial was ignored and the three preceding informative trials were considered.

The mean number of problems on which an appropriate arrangement was made by the 8.0 to 9.5 age group was 0.8 and by the 9.6 to 10.11 groups was $2.7, t(18)=2.94, p<0.01$. Thus when the appropriateness of the rearrangements made by participants is taken into account, the younger age group has a very low level of performance on the level 3 task.

This finding is consonant with an observation which was made during training. Some of the participants appeared to use a strategy of simply changing operators if they were not succeeding on the task. This is a way of achieving a correct arrangement, and thereby reaching criterion for the problem, while circumventing the need to apply a level 3 cognitive system. This strategy would not however result in rearrangements which were appropriate as defined above. Therefore the scores of appropriate rearrangements are probably a truer reflection of the absolute levels of success achieved by the two age groups. On this reasoning success would be virtually negligible for the 8.0-9.5 age group.

\section{Discussion}

The predicted interaction between age and system level is again observed. The point at which children become capable of using level 3 cognitive systems is predictable from memory span requirements derived by Method 1 insofar as it is associated with a digit span of 
six chunks. Experiments 1 and 2 are therefore consistent in showing that memory span requirements of commutativity checking are best predicted by Method 1 .

The finding of the predicted age by level interactions is made more significant by the fact that two recent studies (Brainerd, 1977; Mpiangu \& Gentile, 1975) have shown that interactions predicted by Piagetian theory are not in fact observed. In both cases if was found that the expected interaction between cognitive developmental stage, measured in terms of Piagetian concrete operational performance, and ability to profit from training in further concrete operational tasks was not observed if appropriate tests were used.

The absolute level of performance of the younger group on the level 3 task is fairly high, but this could be achieved partly by the strategy of simply switching operators, as explained previously. When the appropriateness of rearrangements accompanying the switch is taken into account this age group achieved only 0.8 correct problems out of 5 , compared with 2.7 for the older group. Since this measure eliminates correct solutions achieved by switching, it probably provides a fairer indication of the absolute level of performance of the two groups.

\section{RELATION OF THEORY TO EXISTING LITERATURE}

In this section we will consider how the theory relates to certain topics in the contemporary cognitive development literature. We will also consider two topics, conditional discrimination and oddity learning sets, which have not been previously considered within cognitive developmental stage theory, because the current theory is able to generate some new predictions concerning them.

\section{Relationship to Work of Pascual-Leone and Case}

The theory offered differs from that proposed by Pascual-Leone (1970) and Case (1972, 1974) in the amount of information which it regards as necessary for the attainment of each level. A more important difference however is in the way information-processing limitations operate. Case and Pascual-Leone consider that the representations themselves occupy "central computing space," which is equivalent to a type of working memory. That is, working memory must be capable of holding all the chunks necessary to represent the task. The present theory, by contrast, provides for the task elements to be mapped into isomorphs stored in LTM. Demands are made on STM in order to ensure that this mapping process is consistent. Thus STM limitations operate on the consistency checking process, rather than on the representational process as such.

This difference implies that the information-processing limitations postulated by the present theory will operate only where it is necessary to check the consistency of a representation. Where a person already knows how to represent a task, there will be no need to check consistency and the limitation will not operate. Thus the STM limitation will act as a kind of "gate" through which the person must pass when acquiring a new concept, or when first learning to represent a situation. By contrast, Pascual-Leone and Case postulate that central computing space limitations will operate whenever a task is performed. The information processing requirements of a task may be modified by experience (e.g., through chunking), but the limitations continue to operate in some form, whereas in the present theory they do not.

\section{Relationship to Piaget's Theory}

In an overall sense, there is a clear correspondence between Piaget's four major stages and the four levels defined here. Piaget (e.g., 1950) has always insisted that the sensorimotor stage 
differed from later stages in that the former was devoid of symbolic representation. Level 0 as defined here is also a stage without symbolic representation.

In their more recent work Piaget and his collaborators have argued that the preoperational stage is defined in terms of function logic (e.g., Piaget et al., 1968). There are considerable differences between the way they define functions and the mathematical definition given here, and the complex methods used by Piaget et al. to investigate function logic seem in some cases to demand far more than the understanding of a univariate function. Nevertheless it is worth noticing that there is some correspondence between the present definition of level 1 concepts and Piaget's definition of preoperational thought in this respect. Piaget has also argued that preoperational children can cognize one (binary) relation at a time, but cannot coordinate relations (e.g., Piaget, 1950). This also is quite consistent with level 1 thinking as defined in the present theory.

One type of level 1 concept which Piaget does not appear to have considered is the unary operator. Indeed there is one performance, which appears to involve a unary operator, which has often been regarded as an embarrassment to Piagetian theory. This is the finding by Berko (1958) that preschoolers understand elementary grammatical transformations, such as singular-plural, positive-negative, past-present, etc. Although we cannot consider the complex problems of grammatical development here, it is worth noticing that these simple rules, taken alone, can be expressed as unary operators. To this extent then Berko's finding is consistent with the present theory.

Piaget has defined concrete operational thought in terms of his theory of groupings (see Baldwin, 1967, for a lucid explanation). However, Osherson (1974) and Sheppard (1978) have argued convincingly that the theory of groupings is unworkable. However, many of the tasks which Piaget uses as indicators of concrete operational thought really correspond to binary operations. Transitivity, a concrete operational performance, is a partial binary operation as we have already pointed out. Piaget's class inclusion task depends at least partly on disjunctive classification, the truth table for which has the form of a binary operation. The task which Piaget calls multiple classification depends on conjunctive classification, which is another binary operation. Since all concepts which have the form of binary operations are level 2 tasks in terms of this theory, then there is considerable correspondence between level 2 thought and Piagetian concrete operational thought.

It should however be noted that the minimum age at which children are predicted to be capable of acquiring level 2 concepts is 2 to 3 years lower than the age at which Piaget believes children acquire concrete operations. On the other hand the recent literature contains many studies which strongly suggest that children have, or can acquire, concrete operational concepts from the age of 5 years. For instance, Gelman (1978) has suggested that most children used in Piagetian concrete operational training studies probably already have the concept in covert form when training begins. Since most training studies use children between 4.5 and 7 years, this would suggest that concrete operational concepts are available at this age. To the extent that concrete operations involve level 2 reasoning, this would be consistent with our theory.

Piagetian formal operational theory is based partly on the idea of relations between certain logical operations. For instance, Piaget (1957) contends that formal operational children understand the 16 binary operations of propositional logic, and can relate one operation to another in a way which conforms to the INRC group. Each element of the INRC group defines a relation between binary operational forms: Element $I$ defines the relation of each form to itself; Element $N$ relates each element to its inverse; e.g., $N(A \vee B)=\operatorname{not} A \& \operatorname{not} B$; Element $R$ relates each element to its reciprocal; e.g., $R(A \vee B)=$ not $A \vee$ not $B$; Element $C$ defines a relation which corresponds to $N^{*} R$ or $R^{*} N$; e.g., $C(A \vee B)=A \& B$. Each element $I, N$, R, or C 
is equivalent to a unary operator applied to 1 of the 16 binary operations. The application of a set of unary operators to a set of binary operations is tantamount to creation of a system which is equivalent to a ternary operation, which is a level 3 system in our terms. To some extent then basic formal operational thinking corresponds to level 3 as we have defined it.

It should be clear however that our argument provides an entirely different theoretical basis for stages than does Piaget's theory. The levels we have defined do not depend on logicomathematical structures which form part of the inherent structures of the mind, but arise because of the problem of relating representations to segments of the environment in a consistent way. Stage limitations relate to information-processing limitations, rather than to absence of any specific structures. Structure is still important in our theory, but the structures are internal representations of environmental systems, stored in LTM, and acquired through interaction with the environment.

\section{Understanding of Quantitative Relations}

Bullock and Gelman (1977) have elegantly demonstrated that 2.5-to-4-year-old children can represent relations between small sets and can transfer the representation to a new problem. The children were first taught to discriminate between one toy animal and a set of two small animals. They were then tested for transfer to a discrimination between a set of three animals and a set of four animals. The 3-year-olds performed the transfer reliably, as did the 2.5-year-olds under certain conditions. This is a clear example of a level 1 cognitive system, and can be represented as a commutative diagram, as shown in Fig. 23.

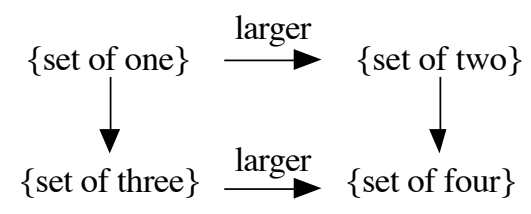

FIGURE 23

The symbol system consists of an internal representation of the original discrimination, which is no longer present in the transfer task, but the essential elements and relationships in which are presumably stored in LTM. The environment system consists of the stimuli, a set of three, and a set of four, which are present in the transfer task. The relation "larger than" exists between the elements of both systems. In order to match the symbol system to the environment system, the symbol element \{set of one\} must be mapped into the environment element \{set of three\}. If this is done then the symbol element \{set of two\} must be mapped into the environment element \{set of four\}. Commutativity can be achieved by mapping only one symbol element into one environment element. Hence the system is level 1 , and it is entirely consistent with the present theory that it should be available to children between 2 and 5 years, as Bullock and Gelman (1977) found.

\section{Transitivity}

So far we have talked in abstract terms about the symbol system used in transitivity, but a considerable amount of recent research has been devoted to the nature of the actual representational process which people employ. Strong evidence has emerged that a common form of representation consists of an ordered array, and the evidence for this has been reviewed by Trabasso $(1975,1977)$. Much of this evidence comes from $n$-term series problems, in which participants are shown a set of stimuli, $a, b, c, d, e$, and are taught that (for instance) $a>b, b>c, c>d, d>e$. Several different lines of evidence converge on the 
conclusion that such a task is represented as an ordered array, $a, b, c, d, e$ (see Trabasso, 1975).

Now we want to relate the ordered array representation to our concept of a symbol system. The first point is that an ordered array conforms to the definition of a system. It consists of a set on which a function is defined, where the function consists of mapping each element in the system into every element smaller than it: $a \rightarrow b, a \rightarrow c, \ldots, d \rightarrow e$. If the system was defined only in terms of relations between each element and every other element, it would be level 1 , but compositions of relations are also defined in the system. For instance, the relation $a R b$ and $b R c$ are defined, but so is the relation $a R c$; therefore it is possible to read off from the system the fact that the relation $a R c$ corresponds to the composition of $a R b$ and $b R c$. A system on which a composition of binary relations is defined is a level 2 system, as defined earlier.

In effect an ordered array incorporates the transitivity principle, because transitivity of an asymmetrical relation is the defining property of an ordered set. Since transitivity is a partial binary operation, it is a level 2 system, so from this point of view too, an ordered array is a level 2 system.

Several points can be made from these considerations. The first is that our characterization of a level 2 system as a set of mappings of the form $S, S \rightarrow S$ or $E, E \rightarrow E$ specifies a general structural property belonging to a class of systems, but there are numerous symbolic and environmental systems which might conform to this definition. On the other hand, once the particular representation used to solve a problem is known, and can be expressed clearly as a set of mappings, there is no difficulty about deciding the level to which it belongs, provided of course only one kind of mapping is involved. For instance, an end-anchor representation of an $n$-term series problem might be expressed as $A \rightarrow a, A \rightarrow b, A \rightarrow e$, where $A$ is the end anchor and $a, b, \ldots, e$, are the task elements. In this case each element is defined by a single binary relation, its relation to the end-anchor, and the system is level 1. It is doubtful whether any adult participants ever use the end-anchor representation exclusively, but there is evidence that it is used in combination with other representations under certain conditions (see Trabasso, 1975).

A further point concerns the age at which the ordered array representation can be used. Trabasso (1975) has argued that the findings of research on $n$-term series representations argue against cognitive developmental stage theory because all age groups from 6 years to adulthood use the same representation. Since however an ordered array is a level 2 system, and should be available to children over 4.5 years, this is precisely what we would expect in terms of the current theory. What we should not expect is that the same representation would be used by children under 4.5 years, because a level 2 system should be beyond the capacity of average children below this age.

There is evidence that 4-year-olds do solve $n$-term series problems, but there is also evidence that they do not use an ordered array representation, but instead use a type of labelling representation (DeBoysson-Bardies \& O'Regan, 1973). More importantly perhaps, most of the studies which have investigated the representation used in $n$-term series problems have used participants over 5 years of age (Trabasso, 1975). What is needed is an extension of this research to children under 4.5. The present theory predicts that evidence will not be found for ability to use ordered array representations in this age range, provided children of superior intelligence are not used.

\section{Seriation}

Seriation is a performance in which children are required to order a set of objects. Transitivity with asymmetry is the defining property of an ordered set. If the ordering relation is already given, then transitivity is the only requirement to be met. Since, as we have already 
shown, transitivity is a level 2 system, then seriation should be a level 2 performance also. Seriation may be thought of therefore as an application of the transitivity concept. On the other hand seriation may be easier, as some evidence suggests that it is (e.g., Murray \& Youniss, 1968; Youniss \& Dennison, 1971; Achenbach \& Weisz, 1975) because the child is aided by an image of the seriated set, as with a set of rods varying in height. Where such imagery is precluded, as with the hidden stick seriation task (Baylor \& Leymoyne, 1975), the age of mastery is considerably higher. At the least, seriation requires the coordination of two relations, and a composition of two relations is a binary operation, and therefore a level 2 system. Furthermore, it appears to be precisely in this area of coordination of relations that preschool children tend to go wrong, which is quite consistent with the view that they can apply a representation of only one relation to the task, as this theory would predict.

\section{Conservation}

While we are wary of seeming to oversimplify the complex and subtle problem of conservation, we would like to indicate how it might be conceptualized within the framework presented here. Basically conservation may be seen as a matter of recognizing that a particular class of transformations (such as pouring liquid from one vessel to another, rearranging elements of a set, etc.) is quantity conserving in character. Furthermore, such recognition must take place in the absence of supportive, direct evidence, since the evidence from the immediate situation is either inadequate or misleading.

There are really three classes of transformations which may be applied to quantities: conserving transformations, which neither add nor remove anything; addition transformations, which increase quantity; subtraction transformations, which decrease quantity. In some cases it is difficult to tell the class to which a transformation belongs from its immediate effects; e.g., a conserving transformation may appear to increase quantity, etc. However an incorrect interpretation of a transformation will lead to inconsistencies in the long term. For example, if we incorrectly conclude that a transformation, which was really conserving in character, increased quantity, then sooner or later we will find that the amount we have is not consistent with its having been increased by that transformation. This is because, if any transformation does in fact increase a quantity, then that quantity must be more on all future occasions than it would have been if that transformation had not been performed. If it is not more, then the transformation cannot have been an addition transformation.

Now we will consider a possible conceptualisation of quantity which would permit such inconsistencies to be detected. Basically, we can consider quantities as collections of units added together. This receives some psychological justification from evidence by Bearison (1969) that teaching children to regard quantity as composed of units tends to induce conservation. Therefore we might conceptualize quantity as a set of units, or elements, on which an addition operation is defined, as shown below.

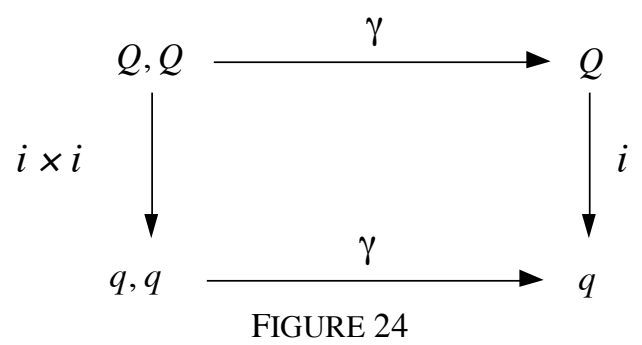

If we consider a quantity dimension consisting of quantities $q_{a}, q_{b}, \ldots, q_{n}$, then we define an addition operation $q_{a}, q_{b}, \rightarrow^{\gamma} q_{a b}$. If we define symbols representing quantities by $Q_{a}, Q_{b}, \ldots, Q_{n}$, 
then recognition that quantities are composed of other quantities added together can be expressed by the addition operation: $Q_{a}, Q_{b}, \rightarrow^{\gamma} Q_{a b}$. The operation $\gamma$ is a stored set of mappings of units into their sums; i.e., it represents the knowledge that quantity is composed of units which combine additively. The interpretation of a quantity-problem situation would then correspond to the diagram in Fig. 24.

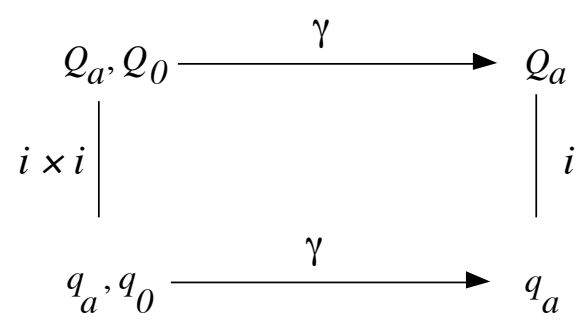

FIGURE 25

A quantity-conserving transformation would be equivalent to adding nothing, so a quantityconserving transformation applied to a quantity $q_{a}$, would be represented by $q_{a} q_{0} \rightarrow^{\gamma} q_{a}$, A conservation performance would then be represented by the diagram in Fig. 25.

A non-conservation performance would correspond to the diagram in Fig. 26. Thus a nonconservation performance corresponds to a diagram which does not commute. In effect the non-commutativity of the cognitive system is equivalent to asserting that there is an inconsistency between quantities as they exist in the environment, i.e., as an additive system of units in which the specific class of transformation $q_{0}$ is conserving, and the person's interpretation of the observed transformation as an addition transformation.

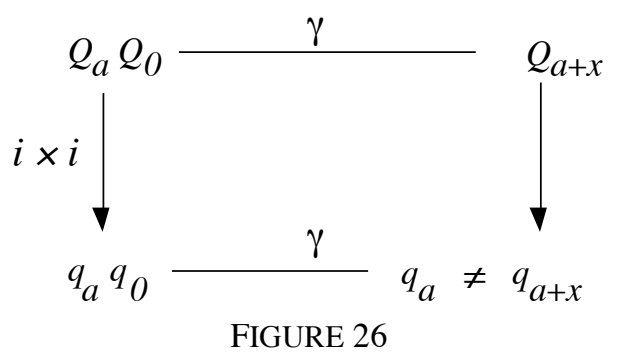

\section{Dimension-Abstracted Oddity Learning Sets}

It is possible to show that, provided certain criteria outlined below are met, the dimensionabstracted oddity learning set task would be level 2 according to the theory. An oddity problem, reduced to its minimal components, has three stimuli, two of which are the same on one dimension and one is different. We will call these $S_{1}, S_{2}$, and $O$. The oddity concept consists of three elements which are defined by their relations to the other elements as follows: $S_{1}$ is the same as $S_{2}$, and vice versa. The odd element, $O$, is defined as different from $S_{1}$ and different from $S_{2}$. Each relation between any element and any other element is a binary relation; that is, the relations same as and different from can be written as sets of ordered pairs. The oddity concept is defined as a composition of these two relations; i.e., it must be composed of a set of stimuli which are the same as other stimuli, plus one stimulus which is different from the others. A problem in which all the stimuli were the same or all different would not be an oddity problem. Since a composition of binary relations is mathematically equivalent to a partial bivariate function, then the oddity concept is level 2 .

If a person has an internal representation of the oddity concept, he/she can decide which is the odd object on the first trial of a new problem. For example, if the three stimuli of a 
problem consist of two circles and a triangle, then triangle can immediately be selected as odd. Notice however that, in this simple problem, it is possible to adopt a simpler mode of solution. If we know in advance that every problem contains two identical stimuli and one different stimulus, then any stimulus which is different from the other two is odd. The choice can be made by considering only one relation, which can be expressed as a set of ordered pairs as follows: $d=\{$ (triangle, circle 1), (triangle, circle 2) $\}$, where $d$ is the relation of difference between the triangle and the two circles. This discrimination is based on a single binary relation, and accordingly would become a level 1 task.

It has become the practice in the literature therefore to use two dimensions in order to force choice on the basis of the oddity principle. This is the familiar dimension-abstracted oddity problem, an example of which could be constructed by using the following stimuli: red circle, blue triangle, green triangle. This prevents selection of the odd object by considering only one difference relation. For instance, green triangle differs from blue triangle in colour, but green triangle is not the odd object in this set. Since all the stimuli differ in colour, this dimension does not define the odd object. However, since two of the stimuli are the same shape and one is different, oddity is defined in terms of the shape dimension, and circle is therefore the odd object in the set. The dimension-abstracted oddity problem therefore forces the participant to select the odd object by considering both the same and difference relations. It therefore requires a level 2 cognitive system, as we will show below.

The oddity concept can be conveniently expressed as three elements, with arrows between them representing the same and difference relations, as shown in Fig. 27. This system can be mapped into either dimension of any oddity problem. If it is mapped into the dimension which defines oddity for that problem, a consistent match is obtained. This is illustrated in Fig. 27a, where the system defining the oddity concept is mapped into the shape dimension in the example given below. If the system is mapped into the irrelevant dimension (i.e., the one which does not define oddity), then no consistent match can be achieved. This is illustrated in Fig. 27b, where the system is matched into the colour dimension of the same example. In this case the relations in the symbol system sometimes correspond to those in the problem, but in other cases they do not, so the mapping is inconsistent and the cognitive system does not commute.

It follows from the argument in this section that correct first-trial performance on a dimension-abstracted oddity learning set task should be subject to the same age constraints as other level 2 tasks; i.e., it should not be achieved before $41 / 2$ years in average children. This specific prediction does not appear to have been tested in the literature. It is perfectly testable however, but there are two precautions which should be observed.

The first is that the relevant dimension must vary from problem to problem in learning set training. If this is not done the irrelevant dimension may be ignored later in training. The result would be that, if participants attend to only the relevant dimension, the odd object can be selected by considering only one relation, as described above for simple, unidimensional oddity problems, which would reduce the task to level 1 . The second precaution is that the practice of placing the odd object on either the left or the right, with the centre stimulus position never being odd, must not be used. The odd object should vary randomly over all three stimulus positions from trial to trial. If the centre position is never odd, it is again possible to adopt a simpler mode of solution. This is because, with such a procedure, it turns out that any stimulus which is different from the centre stimulus is necessarily odd, which means that it can be selected by considering only one relation, again reducing the task to level 1. Provided these precautions are observed, the theory does predict that the minimum age of attainment of the oddity concept, as measured by correct first-trial performance on dimensionabstracted oddity learning set tasks, will be as for other level 2 tasks. 


\section{Conditional Reaction Learning Set}

Another performance which would require a level 2 cognitive system would be first-trial transfer in a conditional reaction learning set. Normally a conditional reaction task consists of two binary stimulus dimensions, with responses assigned to stimuli so that $a_{1}, b_{1} \rightarrow R+$, $a_{1}, b_{2} \rightarrow R-, a_{2}, b_{1} \rightarrow R-$, and $a_{2}, b_{2} \rightarrow R+$. This concept is clearly level 2 since it has the form of a binary operation. Once this scheme has been learned it is possible to perform perfectly on the first trial of a new problem after feedback has been received for one item of that problem. Suppose for instance that the stimulus values of a new problem are red, green, triangle, and square. Once it is found that, for instance, red triangle is positive, it follows that red square and green triangle must be negative, while green square must be positive. In order to demonstrate that the child has the system, first-trial transfer to a new problem would have to be significantly higher than could be achieved with a less complex rule. Selection of an appropriate baseline against which to measure transfer is therefore important.

Gollin and Liss (1962) and Gollin $(1964,1965,1966)$ have studied the conditional reaction problem with children, and found that preschool children had difficulty learning it. Nevertheless with special "help" procedures 3- and 4-year-olds did succeed in some cases, and even showed some transfer. However no baseline was used and the absolute level of transfer is not reported in detail. It does not appear that transfer was sufficient to demonstrate that the children were applying a representation of the conditional reaction concept to the transfer tasks.

\section{CONCLUDING COMMENTS}

We have provided what amounts to a new formulation for cognitive developmental stage theory. The basis of the formulation is that symbolic representations may be defined in terms of three formally distinct levels of systems. We have also argued that the use of symbolic representations requires the internal representation, or symbol system, to be mapped into a problem in a way which is consistent, defined by a commutative diagram. The process of insuring commutativity imposes higher information-processing loads with higher-level systems than with lower-level systems, and we suggest that this factor may limit the highest level of systems which children can use in problem solving. Some empirical work which supports this argument is presented.

In effect the argument defines a dimension of task difficulty, system level, which corresponds in certain respects to those cognitive developmental stages which have already been observed. The theory is not limited however to explaining preexisting phenomena on an ad hoc basis, and we have illustrated this both by means of a new empirical paradigm, and by predicting as yet unobserved constraints on learning in the dimension-abstracted oddity and conditional reaction learning set tasks.

No claim is made that the theory incorporates all the characteristics of preexisting cognitive developmental stage theories. Indeed, in some respects the stages we have defined are decidedly different in character from those of, say, Piaget. One example of this was pointed out earlier when we said that stage transitions would not occur abruptly across a whole population, but there would be a gradual increase in the proportion of children capable of reaching a particular level as age increases. This "gradual" transition is more consistent with the data base than is the Piagetian idea of abrupt transitions, because there is more evidence for gradual than for abrupt transitions (e.g., Flavell, 1977). The theory is still a stage theory however in the sense that formally distinct levels of thought have been defined, the levels are rank ordered with respect to difficulty, and should be attained in a sequence which 
corresponds to this ordering. Furthermore, the approximate minimum age of mastery of each level has been predicted.

Although this article has been addressed primarily to cognitive developmental phenomena, we should mention that there may be implications for the issue of symbolic representations as well. We have provided a criterion, commutativity, for deciding whether a representation is valid or not, and this criterion may find uses in cognitive theory outside the developmental area. Although the issue of whether, and how, symbolic representations are used to mediate behaviour has a long history in psychology, there has been little attempt to base any such arguments on a precise definition of representations. Thus many consequences which logically follow from the use of representations have been overlooked. In this article we have explored one set of such consequences which happen to be relevant to the cognitive development area. There must however be many other consequences which could be derived from representation theory, and which could find application in relation to other cognitive issues. For that matter, the delineation of levels of thought is a concept whose relevance is by no means exclusively developmental. We hinted at wider applications earlier when discussing the work of Baddeley and Hitch (1974) where we pointed out that the fact that level 1 reasoning tasks were used may affect the interpretation of their work. The existence of a dimension of task difficulty which corresponds to levels of thought probably has potential for wider application in investigations of cognitive processes.

\section{REFERENCES}

Achenbach, T. M., \& Weisz, J. R. Impulse-reflectivity and cognitive development in preschoolers: A longitudinal analysis of developmental and trait variance. Developmental Psychology, 1975, 11, 413-414.

Arbib, M. A., \& Manes, E. G. Arrows, structures, and functors: The categorical imperative. New York: Academic Press, 1975.

Baddeley, A. D., \& Hitch, G. Working memory. In G. H. Bower (Ed.), The psychology of learning and motivation: Advances in research and theory. New York: Academic Press, 1974. Vol. 8.

Baldwin, A. L. Theories of child development. New York: Wiley, 1967.

Baylor, G., \& Lemoyne, G. Experiments in sedation with children: Towards an information processing explanation of the horizontal decalage. Canadian Journal of Behavioral Science, 1975, 7, 4-29.

Bearison, D. J. Role of measurement operations in the acquisition of conservation. Developmental Psychology, $1969,1,653-660$.

Berko, J. The child's learning of English morphology. Word, 1958, 14, 150-177.

Brainerd, C. J. Cognitive development and concept learning: An interpretive review. Psychological Bulletin, 1977, 84, 919-939.

Broadbent, D. E. The magic number seven after fifteen years. In A. Kennedy \& A. Wilkes (Eds.), Studies in long term memory. London: Wiley, 1975.

Bruner, J. S. The course of cognitive growth. American Psychologist, 1964, 19, 1-15.

Bryant, P. E., \& Trabasso, T. Transitive inferences and memory in young children. Nature (London), 1971, 232, 456-458.

Bullock, M., \& Gelman, R. Numerical reasoning in young children: The ordering principle. Child Development, $1977,48,427-434$.

Case, R. Validation of a neo-Piagetian mental capacity construct. Journal of Experimental Child Psychology, 1972, 14, 287-302.

Case, R. Strictures and structures: Some functional limitations on the course of cognitive development. Cognitive Psychology, 1974, 6, 544-573.

Cohen, R. L_ \& Sandberg, T. Relation between intelligence and short-term memory. Cognitive Psychology, 1977, 9, 534-554.

Coombs, C. H., Dawes, R. M., \& Tversky, A. Mathematical psychology: An elementary introduction. Englewood Cliffs, NJ: Prentice-Hall, 1970.

DeBoysson-Bardies, B., \& O'Regan, K. What children do in spite of adult's hypotheses. Nature (London), 1973, 246, 531-534.

Dempster, F. N. Memory span and short-term memory capacity: A developmental study. Journal of Experimental Child Psychology, 1978, 26, 419-431. 
Farnham-Diggory, S. The development of equivalence systems. In S. Farnham-Diggory (Ed.), Information processing in children. New York: Academic Press, 1972. Chap. 3.

Flavell, J. H. What is memory development the development of? Human Development, 1971, 14, 225-288.

Flavell, J. H. Cognitive development. Englewood Cliffs, NJ: Prentice-Hall, 1977.

Friedrich, D. Developmental analysis of memory capacity and information-encoding strategy. Developmental Psychology, 1974, 10, 559-563.

Gelman, R. Cognitive development. Annual Review of Psychology, 1978, 29, 297-332.

Gollin, E. S. Reversal learning and conditional discrimination in children. Journal of Comparative and Physiological Psychology, 1964, 58, 441-445.

Gollin, E. S. Factors affecting conditional discrimination in children. Journal of Comparative and Physiological Psychology, 1965, 60, 422-427.

Gollin, E. S. Solution of conditional discrimination problems by young children. Journal of Comparative and Physiological Psychology, 1966, 62, 454-456.

Gollin, E. S. \& Liss, P. Conditional discrimination in children. Journal of Comparative and Physiological Psychology, 1962, 55, 850-855.

Greeno, J. G. The structure of memory and the process of solving problems. In R. S. Solso (Ed.), Contemporary issues in cognitive psychology. New York: Wiley, 1973.

Halford, G. S. Cognitive developmental stages emerging from levels of learning. International Journal of Behavioral Development, 1978, 1, 341-354.

Hitch, G. J. The role of short-term working memory in mental arithmetic. Cognitive Psychology, 1978, 10, 302323.

Hunt, E. Mechanics of verbal ability. Psychological Review, 1978, 85, 109-130.

Hunt, E., Frost, N., \& Lunneborg, C. Individual differences in cognition: A new approach to intelligence. In G. Bower (Ed.), The psychology of learning and motivation. New York: Academic Press, 1973. Vol. 7.

Huttenlocher, L, \& Burke, D. Why does memory span increase with age? Cognitive Psychology, 1976, 8, 1-31.

Inhelder, B., \& Piaget, J. The growth of logical thinking. New York: Basic Books, 1958.

Keating, D. P., \& Bobbit, B. L. Individual and developmental differences in cognitive processing components of mental ability. Child Development, 1978, 49, 155-167.

Kessen, W. "Stage-and-structure" in the study of children. Monographs of the Society for Research in Child Development, 1962, 27, 65-81.

Klahr, D., \& Wallace, J. G. Cognitive development: An information-processing view. Hillsdale, NJ: Erlbaum, 1976.

Lunzer, E. A. Problems of formal reasoning in test situations. In P. H. Mussen (Ed.), European research in cognitive development. Monographs of the Society for Research in Child Development, 1965, 30, 19-46.

Lyon, D. R. Individual differences in immediate serial recall: A matter of mnemonics? Cognitive Psychology, 1977, 9, 403-411.

Luria, A. R. The role of speech in the regulation of normal and abnormal behaviour. London: Pergamon, 1961.

Maclane, S. Categories for the working mathematician. New York: Springer-Verlag, 1971.

Miller, G. A. The magical number seven, plus or minus two: Some limits on our capacity for processing information. Psychological Review, 1956, 63, 81-97.

Mpiangu, B. D., \& Gentile, J. R. Is conservation of number a necessary condition for mathematical understanding? Journal of Research in Mathematics Education, 1975, 6, 179-192.

Murray, J. P., \& Youniss, J. Achievement of inferential transitivity and its relation to serial ordering. Child Development, 1968, 39, 1259-1268.

Newell, A., \& Simon, H. A. Human problem solving. Englewood Cliffs, NJ: Prentice-Hall, 1972.

Osherson, D. N. Logical abilities in children, Vol. 1: Organization of length and class concepts: Empirical consequences of a Piagetian formalism. Potomac, MD: Erlbaum, 1974.

Pascual-Leone, J. A. Mathematical model for the transition rule in Piaget's developmental stages. Acta Psychologica, 1970, 32, 301-345.

Piaget, J. The psychology of intelligence. London: Routledge \& Kegan Paul, 1950.

Piaget, J. The origin of intelligence in the child. London: Routledge \& Kegan Paul, 1953.

Piaget, J. Logic and psychology. New York: Basic Books, 1957.

Piaget, J. Structuralism. New York: Basic Books, 1970.

Piaget, J., Grize, J. B. Szerninska, A., \& Vinh-Bang. Epistemologie et psychologie de la fonction. Paris: Presses Universitaires de France, 1968.

Shepard, R. N., \& Chipman, S. Second-order isomorphism of internal representations: Shapes of states. Cognitive Psychology, 1970, 1, 1-17.

Shepard, R. N., Kilpatrick, D. W., \& Cunningham, J. P. The internal representation of numbers. Cognitive Psychology, 1975, 7, 82-138. 
Sheppard, J. L. From intuitive thought to concrete operations. In J. A. Keats, K. F. Collis, \& G. S. Halford (Eds.), Cognitive development: Research based on a neo-Piagetian approach. London: Wiley, 1978.

Simon, H. A. How big is a chunk? Science, 1974, 183, 482-488.

Suppes, P., \& Zinnes, J. L. Basic measurement theory. In R. D. Luce, R. R. Bush, \& E. Galanter (Eds.), Handbook of mathematical psychology. New York: Wiley, 1963.

Trabasso, T. Representation, memory, and reasoning: How do we make transitive inferences? In A. D. Pick (Ed.), Minnesota symposia on child psychology. Minneapolis: Univ. of Minnesota Press, 1975. Vol. 9.

Trabasso, T. The role of memory as a system in making transitive inferences. In R. V. Kail, Jr. and J. W. Hagen (Eds.), Perspectives on the development of memory and cognition. Hillsdale, NJ: Erlbaum, 1977.

White, S. H. Evidence for a hierarchical arrangement of learning processes. In L. P. Lipsitt \& C. C. Spiker (Eds.), Advances in child development and behavior. New York: Academic Press, 1965.

Youniss, J., \& Dennison, A. Figurative and operative aspects of children's inference. Child Development, 1971, 42, 1837-1847. 